\title{
Angstrom-scale Ruler Using Single Molecule \\ Conductance Signatures
}

James McNeely, ${ }^{1}$ Nicholas Miller, ${ }^{1}$ Xiaoyun Pan,,${ }^{1,3}$ Brent Lawson, ${ }^{2}$ Maria Kamenetska*1,2,3

${ }^{1}$ Department of Chemistry, ${ }^{2}$ Department of Physics, ${ }^{3}$ Division of Material Science and Engineering; Boston University, Boston MA

\section{Supporting Information}

\section{Contents}

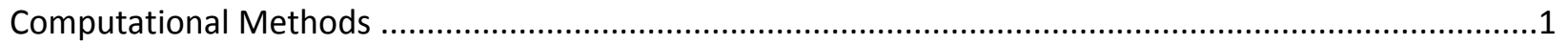

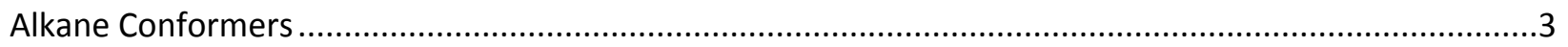

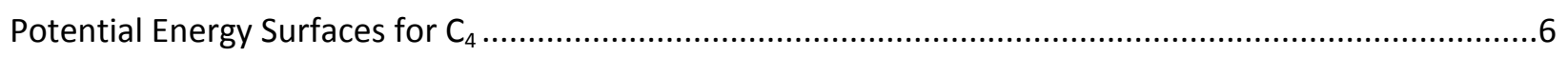

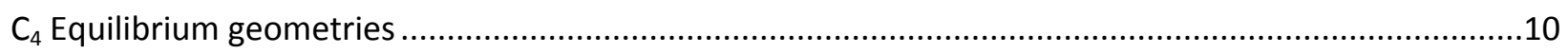

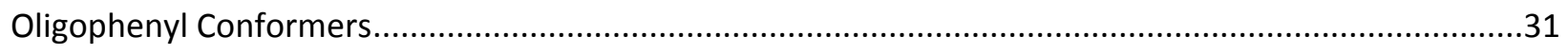

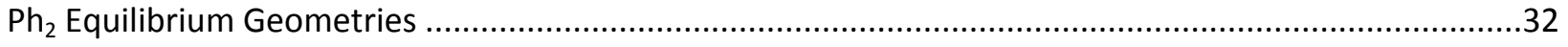

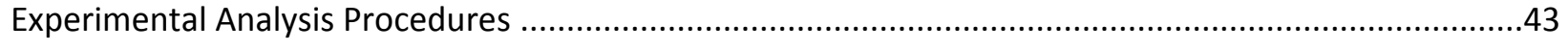

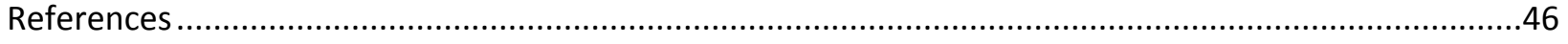

\section{Computational Methods}

All calculations were performed with ORCA, version $4^{1-3}$. The BP86 $6^{4,5}$ functional was used with the DEF2-SV $(\mathrm{P})^{6}$ basis set. Grimme's dispersion correction were applied with Becke-Johnson 
damping $(\mathrm{D} 3 \mathrm{BJ})^{7,8}$, and the $\mathrm{DEF} 2 / \mathrm{J}^{9}$ auxiliary basis set was employed for the evaluation of the coulomb integrals. The compound keyword GRID7 was used for the integration grid.

When generating potential energy surfaces (PES), several constraints were imposed to limit the scans to a single degree of freedom. The two electrodes, modeled as $\mathrm{Au}_{20}$ pyramids (optimized individually at the BP86/DEF2-SV(P)/D3BJ level), were placed such that the gold atom located near the center of mass of the base of each pyramid and the corresponding apex atom were forced to lie on the same vector. The tip-tip geometries for the different conformations of the linker molecule were then varied by moving the pyramids away from one another along this vector. The constraints were applied by fixing the Cartesian coordinates of the four atoms comprising the vector definition.

The suitability of this theoretical model was tested by comparing relative energies and PES minima with calculations performed at the $\mathrm{PBE} 0^{10} / \mathrm{DEF} 2-\mathrm{TZVP} / \mathrm{D} 3 \mathrm{BJ} / \mathrm{RIJCOSX}^{11-13}$ level two of the conformers for $\mathrm{C} 4$-Diamine $\left(\mathrm{C}_{\mathrm{a} 3}\right.$ and $\mathrm{C}_{\mathrm{g} 3}$ as discussed below). As can be seen, the BP86 model severely overestimates the binding energy compared with the PBE0/DEF2-TZVP model. The minima of gauche conformer [SC1(i) and SC1(iii)] are separated by $9.5 \mathrm{kcal} / \mathrm{mol}$, and the anti conformers have a difference in binding energy of $9.4 \mathrm{kcal} / \mathrm{mol}$ at the minimum. The relative binding of the different conformers, however, was well-reproduced in the two models. The relative binding between the gauche and anti conformers at the minimum was $0.3 \mathrm{kcal} / \mathrm{mol}$ with the PBE0/DEF2-TZVP model and $0.5 \mathrm{kcal} / \mathrm{mol}$ with the BP86/DEF2-SV(P) model. It is these relative bindings between conformers that is relevant for the conclusions described in the main text. Furthermore, the location of the minima are well-reproduced with the more economic BP86/DEF2$\mathrm{SV}(\mathrm{P})$ model. 


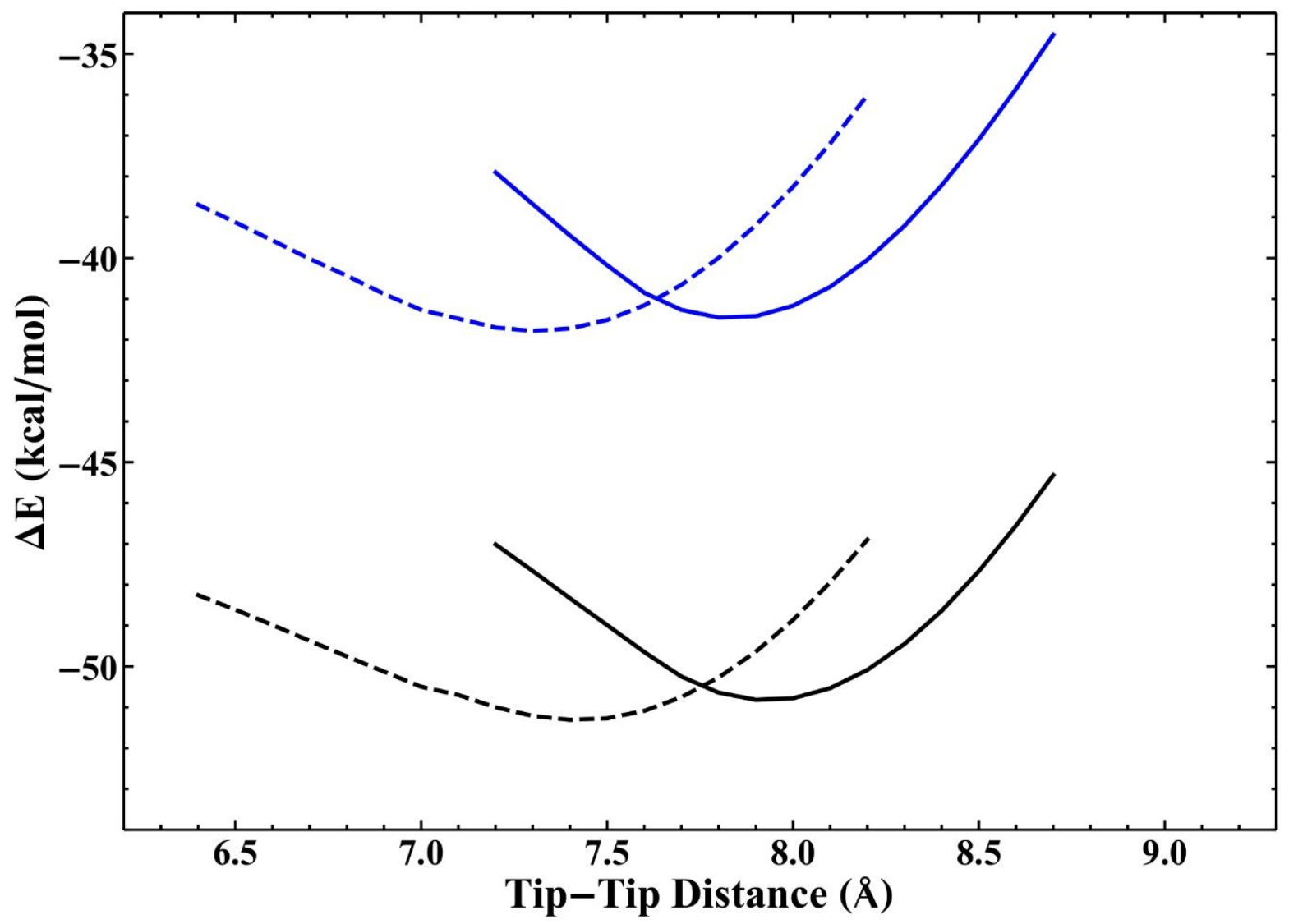

Figure SC1. Binding of gauche conformer at the PBE0/DEF2-TZVP/D3BJ/RIJCOSX (i), anti conformer at the PBE0/DEF2-TZVP/D3BJ/RIJCOSX (ii), gauche conformer at the BP86/DEF2$\mathrm{SV}(\mathrm{P}) / \mathrm{D} 3 \mathrm{BJ}$ (iii), and anti conformer at the BP86/DEF2-SV(P)/D3BJ (iv) levels.

\section{Alkane Conformers}

In the following section, the selection of $\mathrm{C}_{4}$ conformers will be discussed. The conformers that we attempted to locate are shown in Schemes SC1 and SC2 along with the labeling scheme used. In Schemes $\mathrm{SC} 1$ and $\mathrm{SC}$, black labels refer to conformers that were located, while red labels refer to conformers that we were unable to locate. Guided by the snapback data in Figure 1E, we only look for conformations where the gap between the two Au20 pyramids, as defined in Figure 1A, is greater than 4.0 Angstroms. For the relatively short $\mathrm{C}_{4}$ diamine, we found that tip-tip 
binding, where both amines are bound to the apex atoms, is more stable for the gap distances studied experimentally than the corresponding tip-edge and edge-edge binding geometries. It is expected that the edge-tip and edge-edge binding motifs are more prevalent for the longer diamines studied in this work.

For the tip-tip conformations summarized in Scheme SC1, we were able to converge 11 of the 13 conformations for which we searched. For the tip-edge conformations, only 8 of the 13 available conformers were located and converged. The labeling scheme used here consists of the first letter that represents to gauche or anti conformation of the $\mathrm{N}_{-} \mathrm{C}_{4}-\mathrm{N}$ backbone, with anti referring to all $\mathrm{N}-\mathrm{C}$ and $\mathrm{C}-\mathrm{C}$ bonds being anti, and gauche referring to one of the $\mathrm{C}-\mathrm{N}$ bonds being gauche to the the $\mathrm{C}_{2}-\mathrm{C}_{3}$ bond. The two numbers that follow refer to tip-tip (11), tip-edge (12), or edge-edge (22). The final number is just a sequential identifier.

The energetic landscapes for the different conformations are summarized in Figures SC2SC8. Figure SC2 shows the PES for the gauche tip-tip conformations. Inspection of Figure SC2 shows that there are two conformations, G11-2 and G11-8, that appear to be bound more strongly than the rest of the gauche tip-tip conformations. It is for this reason that these two conformations were chosen for the Figure 4 in the main text. In Figure SC3, it can be seen that A11-1 and A114 bind more strongly, and thus these are the two conformations that were included in Figure 4.

Figure SC6 provides clear reasons for the ability of the alkyl diamines to act as molecular rulers. Above $\sim 8 \AA$, the A11-1 conformation has the largest binding, and it should be the predominate conformation based on Boltzmann statistics. With the exception of a small region between $6 \AA$ and $7 \AA$, however, it can be seen that the strongest binding is observed between gauche conformers and the $\mathrm{Au}_{20}$ pyramids. The region between $6 \AA$ and $7 \AA$ is fairly dense, and even though and anti conformer binds strongest, it is still expected that the large density of 
available conformations will still reduce the Boltzmann population of the conducting A11-4 conformation.

The tip-edge PES are shown in Figures SC4 and SC5. Inspection of Figure SC7, which shows all of the located conformers, reveals that below $5 \AA$ the G11-8 conformer has a far stronger binding interaction than any of the tip-edge conformers until the tip-tip distance nears $4 \AA$, which is beyond than the experimentally probed range of tip-tip distances.

Scheme SC1. Tip-tip C4-Diamine conformers that were hunted for and found (black label) along with labeling scheme used. Triangles in the center represent the approximate relative orientation of the $\mathrm{Au}_{20}$ pyramids.

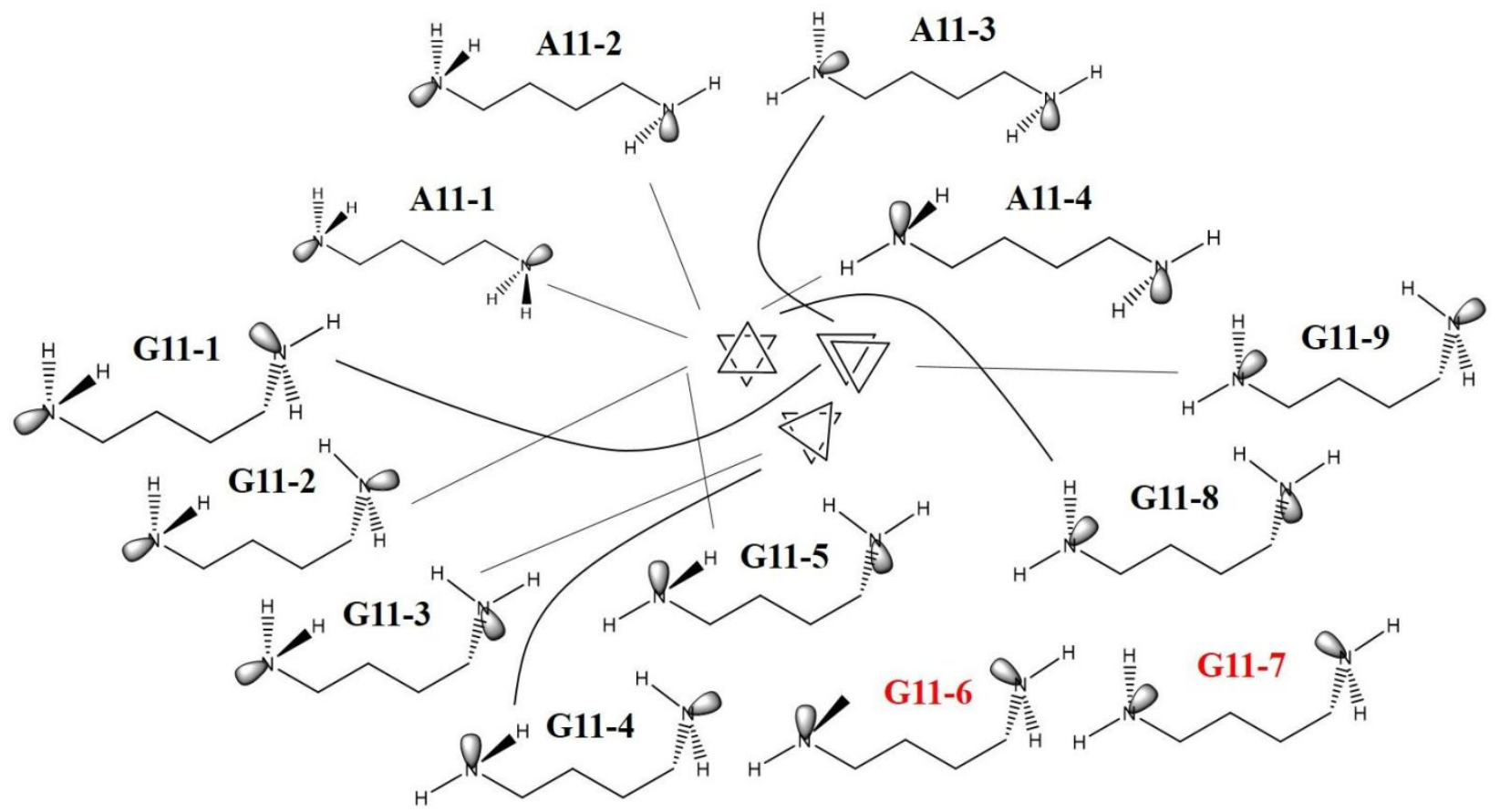

Scheme SC2. Tip-edge C4-Diamine conformers that were hunted for and found (black label) along with labeling scheme used. Triangles in the center represent the approximate relative orientation of the $\mathrm{Au}_{20}$ pyramids. 


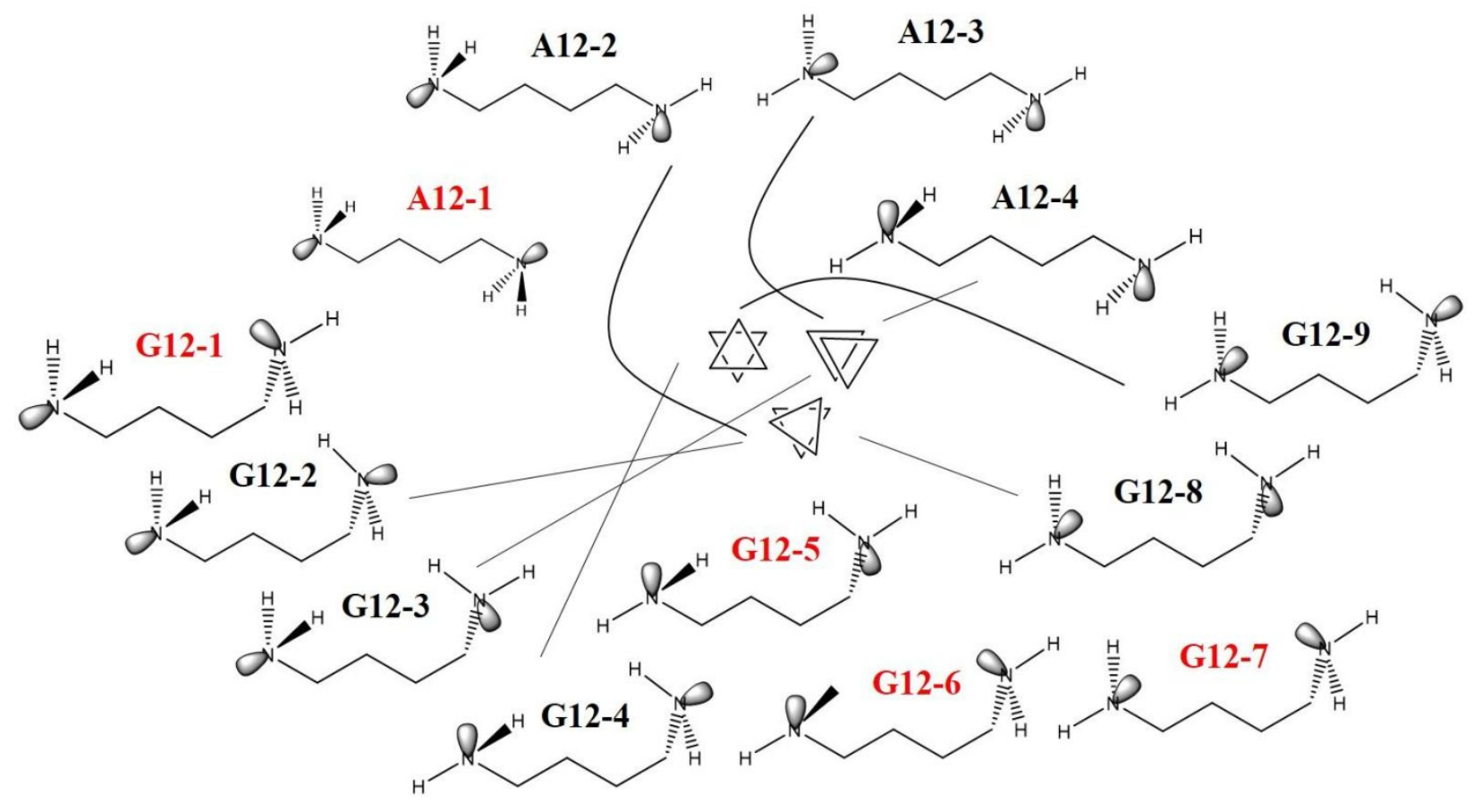

\section{Potential Energy Surfaces for $\mathbf{C}_{4}$}

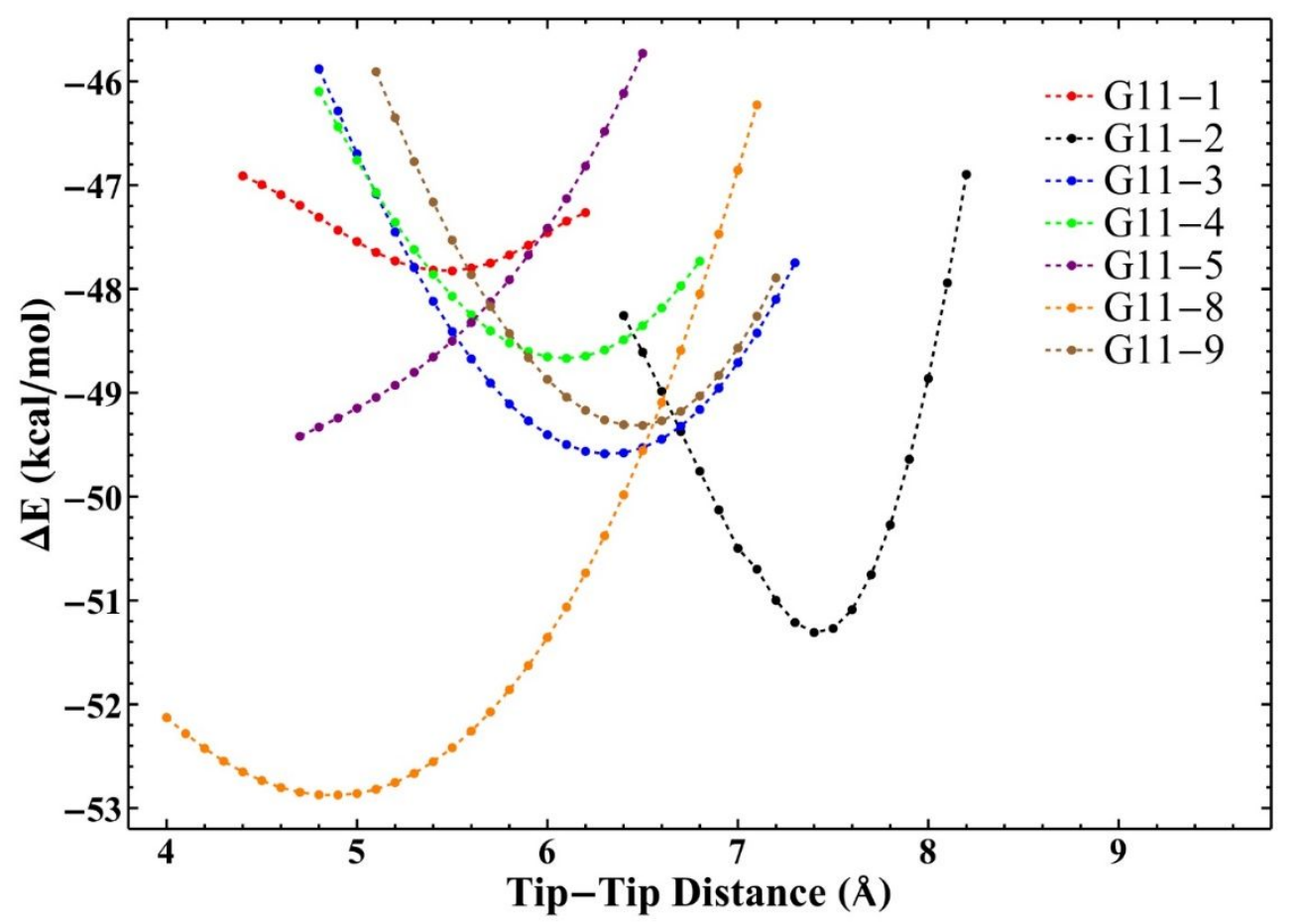

Figure SC2. PES for the gauche tip-tip C4-Diamine conformations calculated at the BP86/DEF2$\mathrm{SV}(\mathrm{P}) / \mathrm{D} 3 \mathrm{BJ}$ level. 


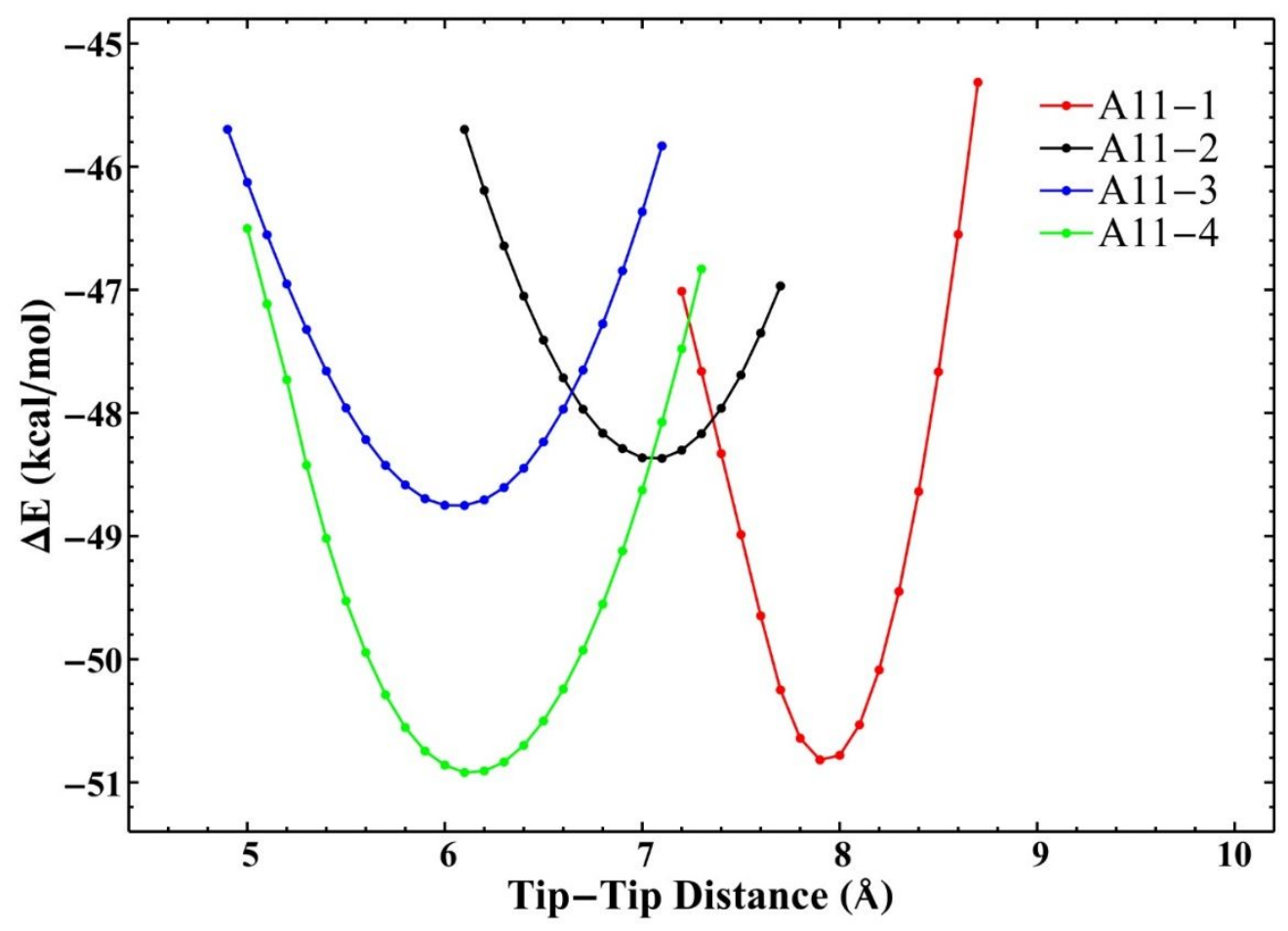

Figure SC3. PES for the anti tip-tip C4-Diamine conformations calculated at the BP86/DEF2$\mathrm{SV}(\mathrm{P}) / \mathrm{D} 3 \mathrm{BJ}$ level.

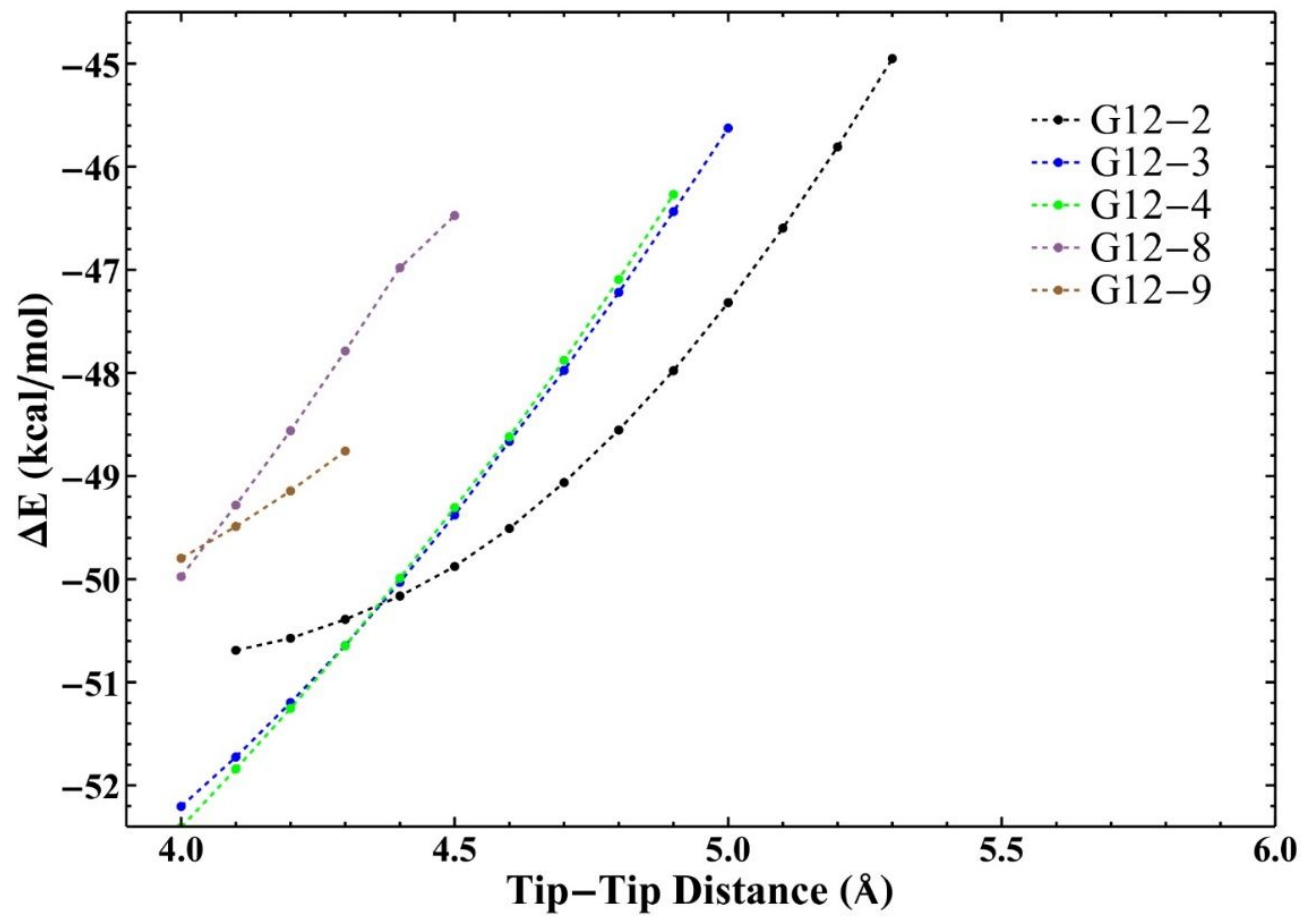

Figure SC4. PES for the gauche tip-edge C4-Diamine conformations calculated at the BP86/DEF2-SV(P)/D3BJ level. 


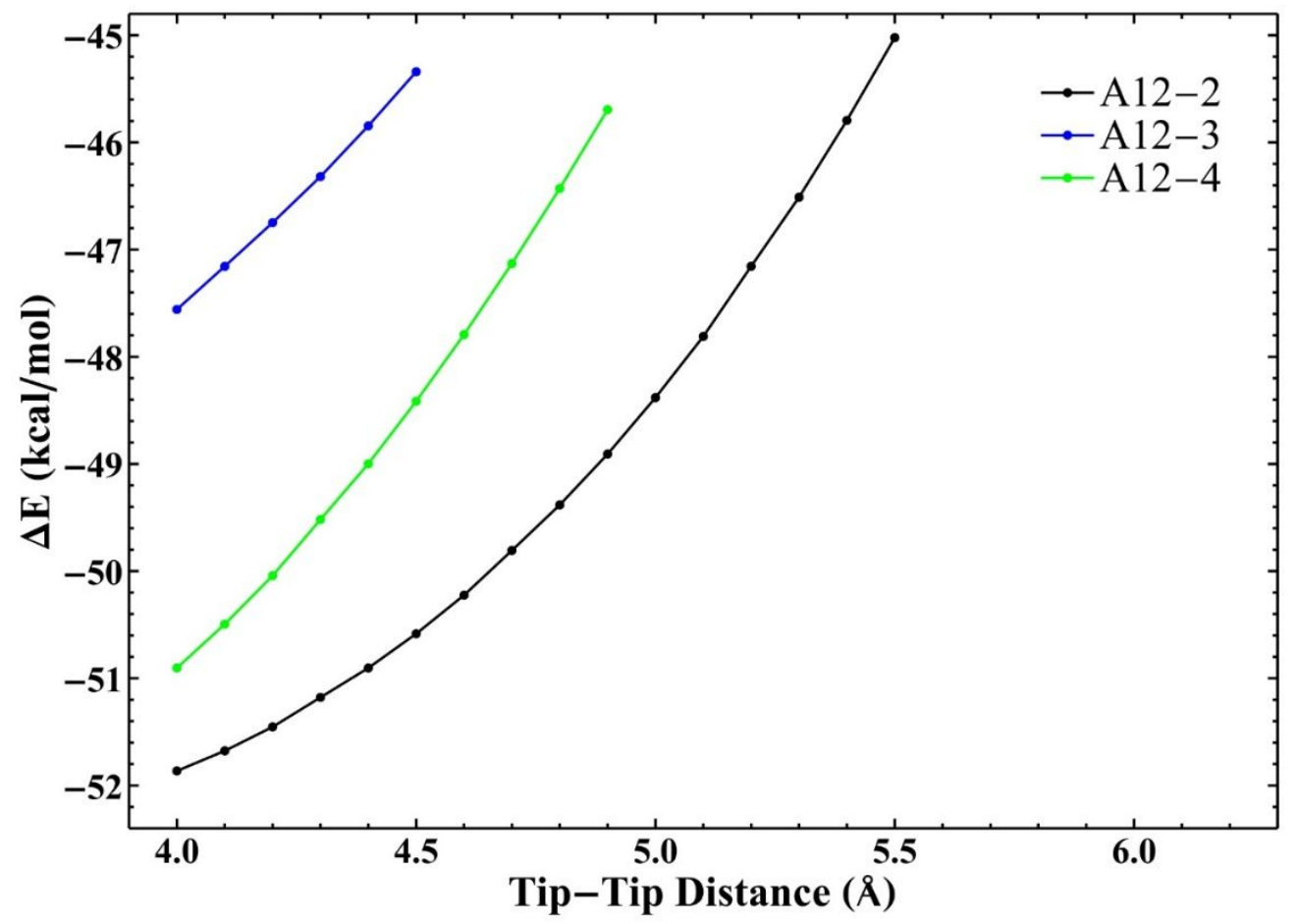

Figure SC5. PES for the anti tip-edge C4-Diamine conformations calculated at the BP86/DEF2SV(P)/D3BJ level.

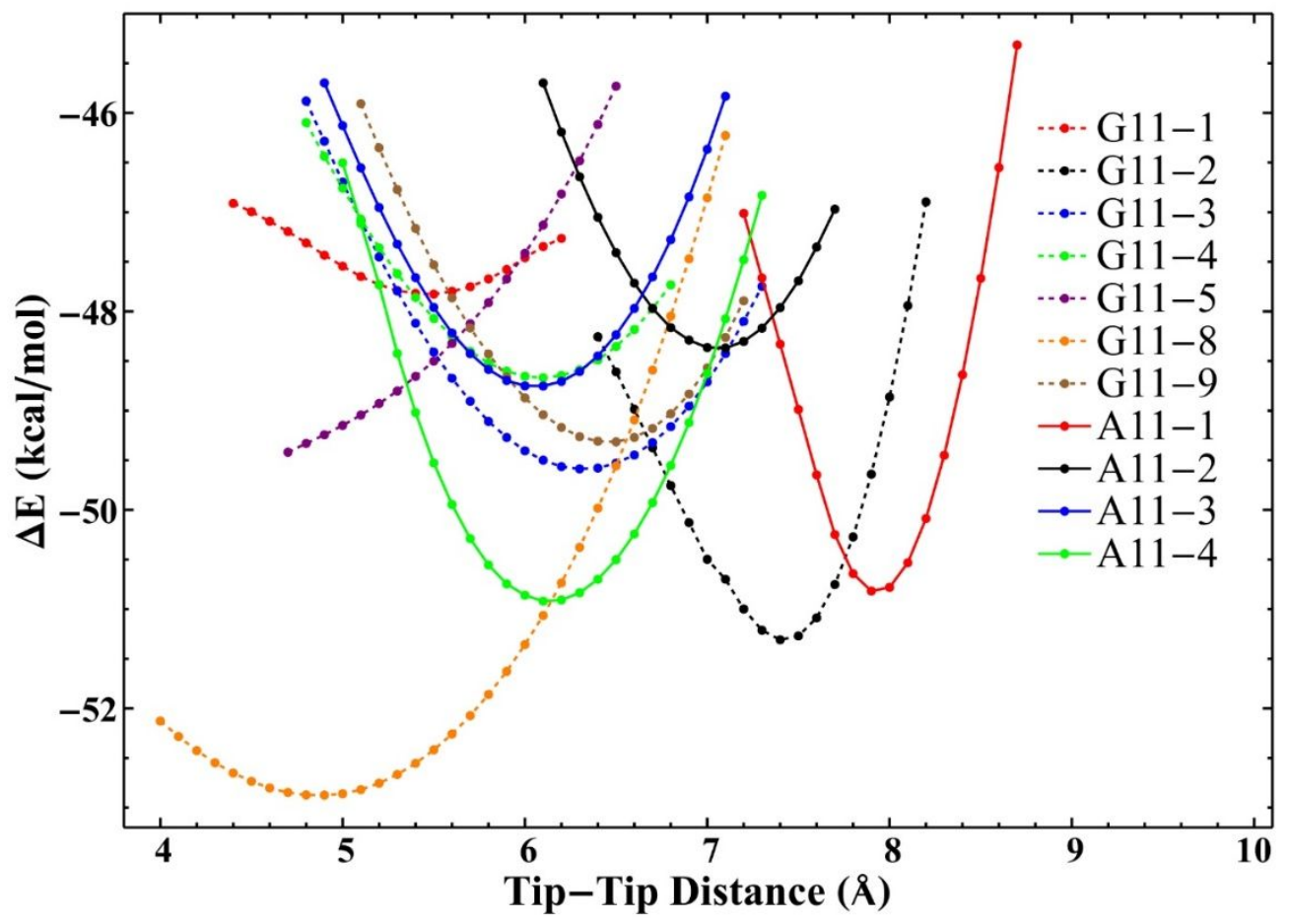

Figure SC6. PES for the gauche and anti tip-tip C4-Diamine conformations calculated at the BP86/DEF2-SV(P)/D3BJ level. 


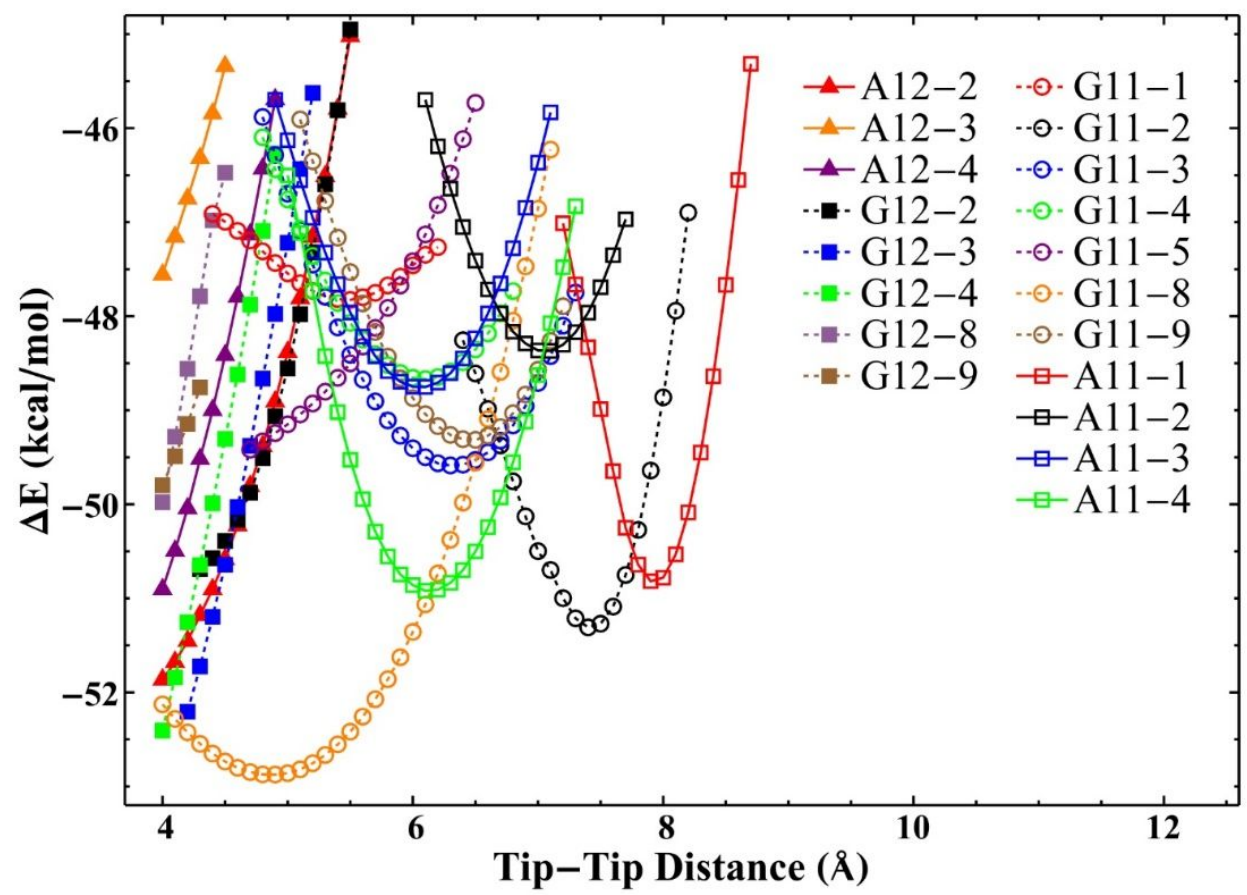

Figure SC7. PES for the gauche and anti tip-edge C4-Diamine conformations calculated at the BP86/DEF2-SV(P)/D3BJ level.

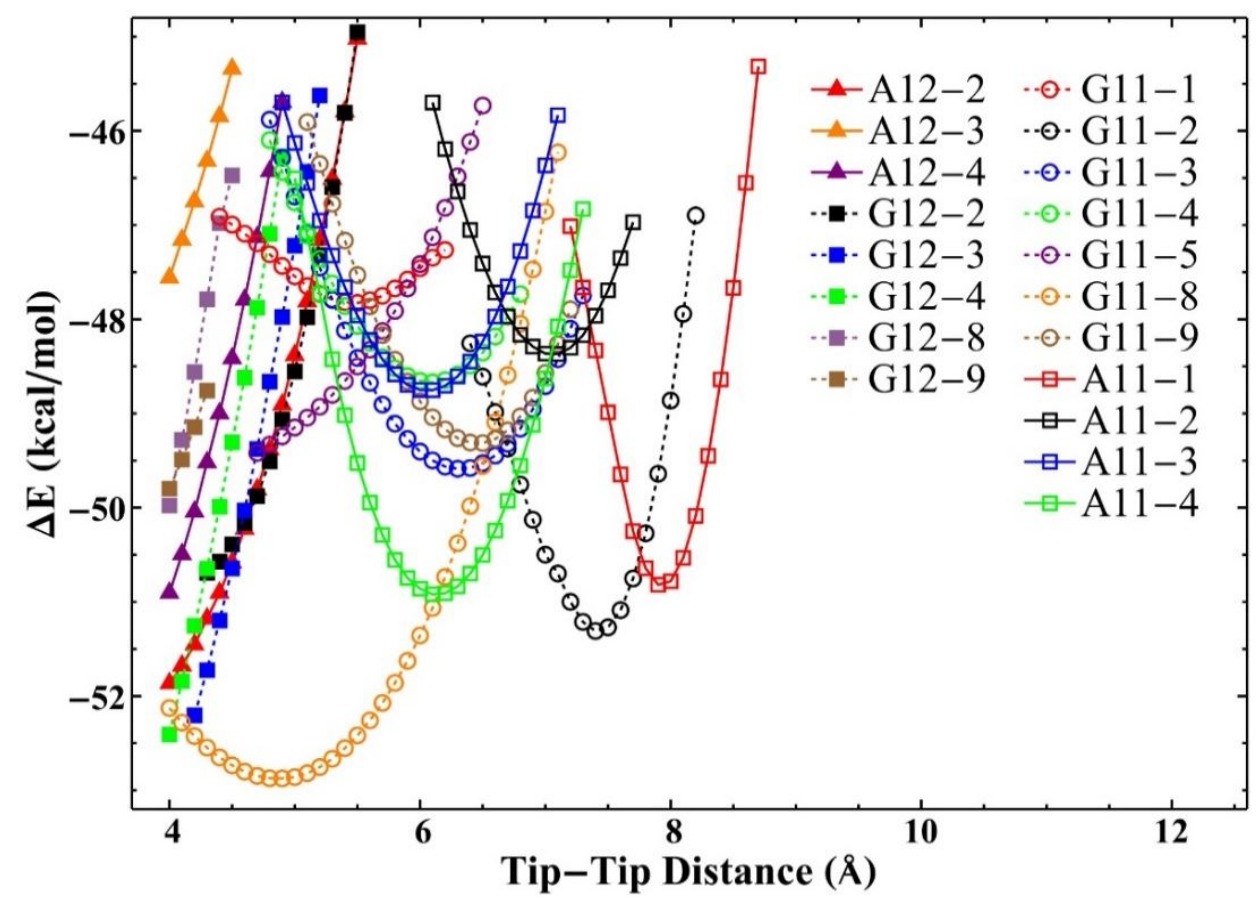

Figure SC8. PES for the all located C4-Diamine conformations calculated at the BP86/DEF2$\mathrm{SV}(\mathrm{P}) / \mathrm{D} 3 \mathrm{BJ}$ level. 


\section{$\mathrm{C}_{4}$ Equilibrium geometries}

Table SC1. Equilibrium geometry for the G11-1 C4-Diamine conformation.

\begin{tabular}{|c|c|c|c|}
\hline $\mathrm{Au}$ & -0.71547075978783 & 2.28415724905568 & -4.36261330652234 \\
\hline $\mathrm{Au}$ & -2.65454176246412 & 3.10957646189624 & -2.59937176747140 \\
\hline $\mathrm{Au}$ & -1.07304134420301 & 0.54756976750409 & -2.24924719226816 \\
\hline $\mathrm{Au}$ & 0.29364273151597 & 3.23605475828670 & -1.99138695819828 \\
\hline $\mathrm{Au}$ & -1.67354745274966 & 4.03133274388773 & -0.08742114991615 \\
\hline $\mathrm{Au}$ & -0.03242610142116 & 1.48670107237330 & 0.23664177160840 \\
\hline $\mathrm{Au}$ & 1.13250725994551 & 4.07970150525755 & 0.42330491483258 \\
\hline $\mathrm{Au}$ & -4.44748382589281 & 3.83698054587206 & -0.72617350724522 \\
\hline $\mathrm{Au}$ & -1.46904714344847 & -0.96030460044348 & -0.05083827920987 \\
\hline $\mathrm{Au}$ & -3.09171377156167 & 1.35792909712761 & -0.39393456829599 \\
\hline $\mathrm{Au}$ & -4.86790320137127 & 2.06947057859660 & 1.71587774585110 \\
\hline $\mathrm{Au}$ & -3.40230931396714 & -0.18516689238936 & 1.99766289247719 \\
\hline $\mathrm{Au}$ & -2.11773211191703 & 2.33857527892126 & 2.40531079300433 \\
\hline $\mathrm{Au}$ & -0.874573 & 36510 & \\
\hline $\mathrm{Au}$ & -6.15931187188647 & 335794948 & 1.31794047667997 \\
\hline $\mathrm{Au}$ & -3.50907912098753 & 4.78191388973649 & 1.96431280275516 \\
\hline $\mathrm{Au}$ & 0.70344144694330 & 2.33008493672385 & 2.86060032472549 \\
\hline $\mathrm{Au}$ & -1.86809145976803 & -2.45094469706367 & 2.24801401125649 \\
\hline $\mathrm{Au}$ & -0.55265608954009 & -0.04044511184883 & 2.59557716229855 \\
\hline $\mathrm{Au}$ & & 4.845 & \\
\hline $\mathrm{Au}$ & -1.28405 & 36654508 & 9388 \\
\hline $\mathrm{Au}$ & -1.50992587002251 & -15.124 & 5139 \\
\hline $\mathrm{Au}$ & -3.22569508242044 & -17.27089971726575 & 4.71533955619475 \\
\hline $\mathrm{Au}$ & -0.31785738501984 & -17.14047231567302 & 5.33480371367572 \\
\hline $\mathrm{Au}$ & -0.52279953858560 & 11618233 & 62415 \\
\hline $\mathrm{Au}$ & -2.28845656369120 & -16.45764598542586 & 623947997 \\
\hline $\mathrm{Au}$ & 0.51648307576613 & -16.27884617746331 & 152753802 \\
\hline $\mathrm{Au}$ & -1.76 & 85649 & 96853 \\
\hline $\mathrm{Au}$ & 449267 & 93105 & 26246 \\
\hline $\mathrm{Au}$ & -3.49728472961913 & -14.49065588832973 & 5.25945017521958 \\
\hline $\mathrm{Au}$ & -3.80176825233593 & -11.80826024840982 & 6.10924207604500 \\
\hline $\mathrm{Au}$ & -5.33375914082914 & -13.65012669263668 & 7.31100340590215 \\
\hline $\mathrm{Au}$ & -2.57644407346821 & -13.4 & 8776623174 \\
\hline $\mathrm{Au}$ & 651 & -13.3885 & 727 \\
\hline $\mathrm{Au}$ & 238 & -9.97 & 930 \\
\hline $\mathrm{Au}$ & -0.8 & -11.6 & 5463 \\
\hline $\mathrm{Au}$ & -1.49264 & -15.4 & 76326 \\
\hline $\mathrm{Au}$ & -6.76306210190864 & -15.65733139204457 & 8.52369046076215 \\
\hline $\mathrm{Au}$ & -4.12124340383501 & -15.55588127105965 & 9.28263240776484 \\
\hline $\mathrm{Au}$ & 1.22751791289946 & -15.28725078215061 & 10.21194145165664 \\
\hline C & -1.39167446878574 & -6.153 & 685709 \\
\hline $\mathrm{H}$ & & -6.76923595436881 & 4.56176368752991 \\
\hline C & -1.50721850054660 & & 4.40117092084687 \\
\hline $\mathrm{H}$ & -2.41553805813647 & -4.23418027648324 & 4.81137640857549 \\
\hline $\mathrm{H}$ & -0.64338246951382 & -4.10739758429346 & 4.72537742592645 \\
\hline $\mathrm{H}$ & -0.45543629341051 & -6.63829868362695 & 4.58984813658829 \\
\hline $\mathrm{N}$ & -1.58912724223035 & -4.62876658528837 & 2.92515459279785 \\
\hline $\mathrm{H}$ & & -4.99836378553258 & 2.4795348118792 \\
\hline $\mathrm{H}$ & .37603261180472 & -5.19044969786587 & 2.56122385849164 \\
\hline $\mathrm{N}$ & -2.52120734069728 & -8.36297842715624 & 6.71021733290875 \\
\hline $\mathrm{H}$ & -2.70446808373917 & -9.11295719880936 & 7.39717346765179 \\
\hline
\end{tabular}


H $\quad-3.39666756922446$

C -1.39580974741759

H -1.42893554388163

H -0.45177252582364

C -1.41552811969707

H -2.32397912052271

H $\quad-0.54729265858901$
$-7.81970965291008$

$-7.51338766765928$

$-7.36729340113829$

$-8.05722149337915$

$-6.14072238778494$

$-5.58895788892620$

$-5.55735378314708$
6.63207584831295

7.16307147373183

8.26817922880549

6.93556683765310

6.49002555949320

6.83434818547325

6.87214187134228

Table SC2. Equilibrium geometry for the G11-2 C4-Diamine conformation.

Au 3.01570432115351

Au 0.95549014100251

Au 1.53307953925559

Au 0.53799019929201

$\mathrm{Au}-1.64673475978828$

$\mathrm{Au}-1.05022394466033$

$\mathrm{Au}-1.95690165044971$

$\mathrm{Au}-1.16802792501120$

$\mathrm{Au}-0.10048553574472$

$\mathrm{Au}-0.61800287234816$

Au -2.95931208845514

Au -2.44116525270667

Au -3.51040446921915

$\mathrm{Au}-4.32259516459906$

Au -3.42864571579858

Au -3.95305556706947

Au -3.74221562461905

$\mathrm{Au}-1.84127877126763$

Au -2.82547704731901

$\mathrm{Au}-4.55267127398684$

$\mathrm{Au}-6.89611337156804$

$\mathrm{Au}-5.43888283301343$

Au -5.01461845120876

$\mathrm{Au}-4.31946641852084$

Au -2.75253070961374

Au -2.31526923688675

Au -1.74021315652968

Au -3.83111967183944

$\mathrm{Au}-3.05625606428183$

Au -3.47508294574814

Au -1.67725763951159

Au -1.29589956503049

Au -0.48293792389509

Au 0.01594618102770

Au -2.12069524199686

Au -1.01290513855511

Au 0.44181102573076

Au -0.95353263080030

Au -0.17847360669127

Au 0.92882119104923

C -1.77072737966434

H $\quad-2.03471732632899$

C -2.09608960315174

H -3.19005526001331

H -1.80413477430651
4.86100792666844 4.41446490005917 2.61668371590969 5.44233782473743 4.92474001718213 3.16722991946125

5.85085943433353

3.91210292624409

0.54325379862599

2.10089942278001

1.54468348531066 $-0.01349783454226$

2.59078123568516

5.29577288629527

3.33200081797962

4.38232716552115

3.46078129410202

$-1.55753762328782$ 0.95666984377861

6.09726318367288

$-17.43619055796626$

$-15.14477919056722$

$-16.92668829452664$

$-17.93694980524436$

$-15.61213579964512$

$-17.35105275290380$

$-18.26690737237271$

$-13.01872081030124$

$-16.35430776488046$

$-14.56291229499929$

$-12.38670413376436$

$-13.92376437041037$

$-14.95800566566188$

$-15.82735530661515$

$-10.86778165104824$

$-13.35409115386561$

$-17.63694077473963$

$-15.69563923405244$

$-16.73286027882003$

$-18.43374331873927$

$-5.72954669975853$

$-6.35568597142723$

$-4.26631217852684$

$-4.13169190397799$

$-3.60168638354189$
0.30651887077038

$-1.45671060631863$

0.90424359634214

1.34618714482960

$-0.40929777476019$

2.00379335788734

2.27257896015868

$-3.03321492931048$

1.42836514809842

$-0.90290715429110$

$-2.43025443614890$

$-0.29129575836842$

0.17246441504229

0.51092496144757

$-4.47997239445739$

$-1.99651718770370$

2.87433127035499

1.92613745834794

2.42054369593005

3.12435589239500

6.49176204977578

6.02028421015155

8.42960715086748

5.67603879615309

5.15646748131003

7.61622267880637

4.97555545058811

5.64217592133052

10.18525045264977

8.01207971084292

7.54798748110544

9.73238615272926

7.19763511193073

4.52915227943996

5.28043063591726

4.89029200450154

6.94129987505431

11.82415384818622

9.40141431296141

4.36044275328039

3.99508328898501

3.10858090386302

3.66668407248850

3.52260107969137

4.50831466908218 
H -0.66962211522294

N -1.42237986119124

H -0.39626083027358

H -1.68688097084952

N -2.49139269314231

H -3.49086989845025

H -1.95356800478209

C -2.16048514020135

H -2.69703131420198

H -1.07468520696724

C -2.51062124603315

H -3.61272476222797

H -2.27204624638982
$-5.83954092975707$

$-3.76214317131569$

$-3.83893810842994$

$-4.32270666264937$

$-8.70064466711011$

$-8.65188432102723$

$-8.54726875581287$

$-7.68277264405673$

$-7.96743273106353$

$-7.77897256996867$

$-6.23969693870117$

$-6.15422141814003$

$-5.58383463469911$
4.14398701075792

2.44787416800186

2.53720763543885

1.62174921411800

4.60555389674971

4.34899499236695

3.73853699824995

5.63344033867238

6.56298626385806

5.85137894825688

5.24149075736254

5.08679843734899

6.11022713085373

Table SC3. Equilibrium geometry for the G11-3 C4-Diamine conformation.

Au 2.73736455561039

Au 1.13689040569914

Au 1.33933039629146

Au 0.05917235325131

Au -1.66225789654227

Au -1.45105964901031

Au -2.59920211151899

$\mathrm{Au}-0.55848528783414$

Au -0.21254595959717

Au -0.33111343628219

Au -2.24980144573407

$\mathrm{Au}-2.07063830537438$

$\mathrm{Au}-3.41534124249560$

Au -4.49215896599360

Au -2.41344913515778

Au -3.53437381441348

$\mathrm{Au}-4.28124339324907$

Au -1.85680191471005

Au -3.08740805753191

Au -5.32648718930810

Au -7.01850066172921

$\mathrm{Au}-5.52473329937803$

Au -4.67611698372750

Au -4.78754053236525

Au -3.21493632298262

Au -2.29974608987597

$\mathrm{Au}-2.51369527161287$

Au -3.88225049080844

Au -2.30309586894953

Au -3.10262955931600

Au -1.28712216240033

Au -0.50347711756806

Au -0.45406317267363

$\mathrm{Au}-0.71156276498137$

$\mathrm{Au}-2.10517208764413$

Au -1.39414353927938

Au 0.16542063742103

Au 0.19678632651297

Au 0.26231304496593
4.70449342331287

4.10402195690338

2.34621143979313

5.09433778420753

4.40750407859364

2.70461602754547

5.30767521968695

3.43999341825331

0.15276515745074

1.67393964129900

0.94826735636327

$-0.57403093707925$

1.92944054890894

4.56096711599700

2.69108831802990

3.68405895179856

2.77741390822139

$-2.07477343697617$

0.35479152810871

5.34499044745919

$-16.44916889487870$

$-14.25835548532337$

$-16.16360365576242$

$-17.16928862788549$

$-14.96879103383500$

$-16.83381666413365$

$-17.71019966979954$

$-12.23256705677770$

$-15.79189095753950$

$-13.93466703910273$

$-11.90875143160373$

$-13.54201111299400$

$-14.54982636057153$

$-15.47332877043743$

$-10.35054598224977$

$-12.93477034260869$

$-17.29744635907621$

$-15.35993017917360$

$-16.38878380927766$
1.46481199269917

$-0.68562388967053$

1.77205105000300

1.94373076822718

$-0.22294809707504$

2.29166816439377

2.31779768725747

$-2.66723523544022$

1.97831572228987

$-0.43811157402159$

$-2.42441104219496$

$-0.21168001663106$

$-0.00024899904755$

0.11315731551699

$-4.55211686352528$

$-2.24572923830785$

2.57195916205126

2.11248709586022

2.37257029040891

2.60529574094053

7.47901282012922

6.63604554955236

8.86954445272350

6.06282686114391

5.17384693112812

7.42987295023736

4.73139547563555

5.84731961027139

10.07960525491099

8.05397337580485

7.23335166367672

9.21457028121680

6.54447383702710

3.85382177161593

5.09408105458061

4.39442235601843

6.07516058930541

11.15311882505148

8.60406278775506 
$\begin{array}{ll}\text { Au } & -0.10189300624956 \\ \text { C } & -0.84250264269498 \\ \text { H } & -1.39638765739801 \\ \text { C } & -1.45018540046814 \\ \text { H } & -2.49656279560048 \\ H & -0.87494411030736 \\ H & 0.20994782597492 \\ \text { N } & -1.45933333872836 \\ H & -0.53176928193145 \\ H & -2.12989068112167 \\ \text { N } & -0.18642312021003 \\ \text { H } & -0.15748949085120 \\ \text { H } & 0.51166952602360 \\ \text { C } & 0.12632719305654 \\ \text { H } & 0.06751066853405 \\ \text { H } & 1.17198304457082 \\ \text { C } & -0.84704210770899 \\ \text { H } & -1.87605172970712 \\ \text { H } & -0.55827610426743\end{array}$
$-18.12435544098391$ $-6.25684097496681$ $-6.94296913367856$ $-4.85241972955265$ $-4.83477238897616$ $-4.13830721488254$ $-6.20837075895824$ $-4.32546126571682$ $-4.44983875373722$ $-4.84508042209553$ $-9.11436224064805$ $-8.82688179939119$ $-9.86772199319109$ $-7.98451607784223$ $-8.37017487962620$ $-7.62640967405066$ $-6.81969980429854$ $-7.14592141794543$ $-6.01930203150020$
3.46592040536927

3.85796818390922

3.17364550068098

3.74964726766897

4.12295264377669

4.37803848654169

3.48362430196356

2.36385463972252

1.92571047491677

1.77516464832946

4.57577486645393

3.58492101718342

4.68287782026039

5.47887557285140

6.51919341643423

5.31284352812469

5.29077161957360

5.57057907470543

6.00919646663725

Table SC4. Equilibrium geometry for the G11-4 C4-Diamine conformation.

Au 2.26456895295606

Au -0.46658118860291

Au 1.01290572730928

Au 1.18776918177475

Au -1.66507895114490

Au -0.10830531701686

Au 0.00448711749819

Au -3.15038627843797

$\mathrm{Au}-0.35910917955130$

$\mathrm{Au}-1.80548407832876$

Au -4.66341763974186

Au -3.32332545338655

$\mathrm{Au}-3.20858903345470$

$\mathrm{Au}-3.00863114069355$

$\mathrm{Au}-5.88636015699116$

$\mathrm{Au}-4.50300706156976$

$\mathrm{Au}-1.49664448781188$

Au -1.85962299008706

$\mathrm{Au}-1.64112357277060$

Au -1.34621328342035

$\mathrm{Au}-4.94247015051727$

Au -4.07199940770803

Au -4.89977591623964

Au - 2.41797331942758

Au -1.44885217853967

Au -2.29692606688258

Au 0.00691624662456

Au -3.09954950856589

$\mathrm{Au}-4.70498436758042$

Au -4.02523783749734

Au -2.91568992840193

Au -3.65782928224348

Au -1.21682151972390
3.11790436276761

3.32698398635185

1.17356077379992

4.12818689171687

4.31346235848929

2.18077267414664

4.97001671290843

3.44718594259070

$-0.58333860892224$

1.36272803472299

1.50695704680882

$-0.35757185458549$

2.33401477299042

5.11183192806227

3.53319633022348

4.39380040418776

3.02585144785810

$-2.16881524830005$

0.44500017017367

5.71515813629902

$-17.82060442077648$

$-15.20859684763160$

$-17.01703316719430$

$-17.65950000157993$

$-15.00694933514947$

$-16.73977631976413$

$-17.32881639483043$

$-12.73543982524015$

$-16.11308261474006$

$-14.33748598110603$

$-11.77114156930867$

$-13.31647722551985$

$-13.94524406492400$
$-2.54937731822891$
$-2.59095337983001$
$-1.00479155166273$
$-0.24183274486598$
$-0.18906400808580$
1.38419592357236
2.02218198135851
$-2.45673053053217$
0.56173952424132
$-1.02211830697976$
$-0.73920330095664$
0.64958475489905
1.57672293093696
2.19861805731263
$-2.13614727978171$
0.08065671134610
3.75472184711308
2.14637123344125
3.03100563381828
4.30559272945539
3.63881215371236
3.95852985062632
6.26037974916018
4.69797695403735
5.04478059299490
7.39598901371896
5.82413239319582
4.47986233048918
8.78911995945791
6.66523962554329
7.23716610955076
9.31298858805020
7.97372164708002 
Au $1.05314232923998 \quad-14.52891779779898$

Au -2.10235199682305 -10.25650377946067

Au $-0.47087250791209-12.40616335100587$

Au $0.22828386076522 \quad-16.30947698318399$

$\mathrm{Au}-4.34959775039177 \quad-15.08241694283985$

Au -1.99728001904251 -15.73643001727134

Au $2.40937118580517-16.82336940852793$

C $-2.89638818762318-5.08394177176030$

H $-3.80208187416538-5.60146722430665$

C $-3.22850060948326-3.60468370016980$

H $-4.07328135493407 \quad-3.51093490029121$

H $-2.36081692015498 \quad-3.06255418188537$

H $-2.10417919247923 \quad-5.17483328388683$

N $-3.56129580013265 \quad-2.95535630459791$

H $-4.26540751028954-3.50022002943731$

H $-3.97488954068310 \quad-2.02229872786931$

N $-1.80819188591203 \quad-7.97788955608365$

H $-2.08806040523941 \quad-7.65419071762050$

H $\quad-0.79824354912342 \quad-7.78243320929452$

C $-2.58541999458788-7.28917565702676$

H $-3.64895025113732 \quad-7.58402924447768$

H $\quad-2.26350350472489-7.71618245980487$

C $-2.45821794863158-5.76100392547936$

H $-3.09018266712075 \quad-5.39098237581369$

H $\quad-1.41278899570795 \quad-5.46426969405419$
6.34888615491619

5.06019674336165

5.68974433702449

8.65036481620288

11.31425010586712

10.05016577717257

7.06052622009345

4.68407250535716

4.28216067194311

4.87770104150452

5.60238924329451

5.31378039356424

3.90391460652100

3.59313141704348

3.06913469481717

3.75319560919063

4.91993609258461

3.98032554891309

5.01376455906497

5.98009650048451

5.84411200432902

6.95335968553859

5.99460713738872

6.83518503296158

6.24489349464664

Table SC5. Lowest-energy geometry from PES of the G11-5 C4-Diamine conformation.

$\begin{array}{lccc}\mathrm{Au} & -1.67852738432003 & 1.85139182647640 & -4.33159910711533 \\ \mathrm{Au} & -3.17129462080932 & 2.79655418697879 & -2.23814758129272 \\ \mathrm{Au}-1.75349675288200 & 0.08982702029508 & -2.18044774877100 \\ \mathrm{Au}-0.18974409297498 & 2.71642517495056 & -2.20099587365727 \\ \mathrm{Au}-1.68483595761256 & 3.65516239868662 & 0.04810774271087 \\ \mathrm{Au}-0.22840154712683 & 0.95558774734808 & 0.03509593844299 \\ \mathrm{Au} & 1.15392205647171 & 3.46452853192444 & 0.00550574294994 \\ \mathrm{Au}-4.52985020171786 & 3.61190761447913 & -0.06508535886155 \\ \mathrm{Au}-1.82476465759465 & -1.46039521559089 & 0.05715454184334 \\ \mathrm{Au}-3.28736704940624 & 1.03402476691881 & -0.00437336135581 \\ \mathrm{Au}-4.61696236318541 & 1.88612462639055 & 2.39606000018782 \\ \mathrm{Au}-3.33596534210884 & -0.47312131130732 & 2.40451655239579 \\ \mathrm{Au}-1.76654717270954 & 1.90493092418136 & 2.56489544663784 \\ \mathrm{Au}-0.35497653347172 & 4.38534318098068 & 2.46205199705658 \\ \mathrm{Au}-5.78761682862224 & 4.37012767215819 & 2.26540584740416 \\ \mathrm{Au}-3.04372583092738 & 4.45248694447721 & 2.42689509096258 \\ \mathrm{Au} & 1.08395665267366 & 1.74695571457250 & 2.46690001478048 \\ \mathrm{Au}-1.87938000819788 & -2.82711556413048 & 2.38354310170002 \\ \mathrm{Au}-0.31541428257297 & -0.54344441163679 & 2.44339174409251 \\ \mathrm{Au} & 2.38462837779646 & 4.16577674570258 & 2.36838989712288 \\ \mathrm{Au}-1.20620419253102 & -17.49088410563380 & 2.79069588800276 \\ \mathrm{Au}-1.46017433342139 & -14.80060802028713 & 3.30220064583857 \\ \mathrm{Au}-3.20022002085241 & -16.89151029418036 & 4.58589496016762 \\ \mathrm{Au}-0.25807951876682 & -16.74358818820352 & 5.26500255621355 \\ \mathrm{Au}-0.49085320416685 & -13.95829824368260 & 5.87135561368278 \\ \mathrm{Au}-2.27716906542844 & -16.08147642470994 & 7.15881728675868 \\ \mathrm{Au} & 0.52236597738452 & -15.89204658700200 & 7.69566015119093\end{array}$




$\begin{array}{llll}\mathrm{Au} & -1.73052138881408 & -12.21921797590414 & 3.99736763428457 \\ \mathrm{Au} & -5.03200796987875 & -16.17251593772892 & 6.41942236628242 \\ \mathrm{Au}-3.51447735389783 & -14.11188920254576 & 5.16086672797289 \\ \mathrm{Au}-3.78400488544316 & -11.41064346802438 & 6.04552046761758 \\ \mathrm{Au}-5.34463954832538 & -13.27478175038494 & 7.18663433921665 \\ \mathrm{Au}-2.58490252401257 & -13.13400629096677 & 7.96634161531471 \\ \mathrm{Au} & 0.20011070423510 & -12.94181125483419 & 8.42203711135472 \\ \mathrm{Au} & -2.08259497899046 & -9.59820341475706 & 4.82302484068296 \\ \mathrm{Au} & -0.89867447046232 & -11.20569936042597 & 6.63782041616099 \\ \mathrm{Au} & -1.51683985321264 & -15.09811290029890 & 9.73095945603859 \\ \mathrm{Au} & -6.77732740248134 & -15.30714449801518 & 8.35973591529569 \\ \mathrm{Au} & -4.13793063112748 & -15.22801098416593 & 9.14020112412895 \\ \mathrm{Au} & 1.18075798478690 & -14.88439926507895 & 10.15639437044529 \\ \mathrm{C} & -3.39002048577551 & -5.39398200640653 & 4.34829592803172 \\ \mathrm{H} & -4.20701488808579 & -4.65460864172636 & 4.18203163197674 \\ \mathrm{C} & -2.28416522274169 & -4.76127955278287 & 5.19546664126877 \\ \mathrm{H} & -2.60802733967182 & -4.69496612662721 & 6.26220290932115 \\ \mathrm{H} & -1.38268745889990 & -5.41140324390265 & 5.17313316881301 \\ \mathrm{H} & -2.96312242668092 & -5.59080637349528 & 3.33489535277136 \\ \mathrm{~N} & -1.89991077295236 & -3.43111545693802 & 4.67817222816575 \\ \mathrm{H} & -2.57432090915792 & -2.70920109603391 & 4.98112580898226 \\ \mathrm{H} & -0.98054809114703 & -3.13354293475761 & 5.03959798896625 \\ \mathrm{~N} & -3.31488443737268 & -8.34813528765953 & 3.28386032746792 \\ \mathrm{H} & -3.63692778062781 & -9.04662584890680 & 2.59602728614007 \\ \mathrm{H} & -2.74683541264134 & -7.65963500316405 & 2.76444282891266 \\ \mathrm{C} & -4.46250692458977 & -7.70133273224621 & 3.96682990281011 \\ \mathrm{H} & -5.15066614569104 & -7.22148319611620 & 3.23002114140770 \\ \mathrm{H} & -5.02522250620795 & -8.50864052912746 & 4.48274366752103 \\ \mathrm{C} & -3.96317773068013 & -6.67590937766663 & 4.98537935622698 \\ \mathrm{H} & -4.79150573098149 & -6.41623542985730 & 5.68016081693575 \\ \mathrm{H} & -3.18456687400696 & -7.18247337590765 & 5.60928121979823\end{array}$

Table SC6. Equilibrium geometry for the G11-8 C4-Diamine conformation.

$\begin{array}{llrr}\text { Au } & 2.96251513937238 & 3.71268148171307 & 0.84439561299634 \\ \mathrm{Au} & 0.94300467474219 & 3.24336559755186 & -0.96682561728425 \\ \mathrm{Au} 1.48851130791038 & 1.45884285123506 & 1.40086465439181 \\ \mathrm{Au} 0.45576451606950 & 4.25592972895116 & 1.83335107965215 \\ \mathrm{Au}-1.68201353632533 & 3.74920518603853 & 0.01422609067271 \\ \mathrm{Au}-1.12466993799508 & 1.95844202827123 & 2.43256887534485 \\ \mathrm{Au}-2.06255421167869 & 4.64210776867989 & 2.69445454860825 \\ \mathrm{Au}-1.13414671173375 & 2.72090868358194 & -2.59416132900527 \\ \mathrm{Au}-0.11183074411749 & -0.64870446522579 & 1.86370389387428 \\ \mathrm{Au}-0.62917191750379 & 0.91070822821132 & -0.45672101392676 \\ \mathrm{Au}-2.90160926124128 & 0.29502531450318 & -2.02607534043783 \\ \mathrm{Au}-2.40357704871971 & -1.28800968125362 & 0.13145568268460 \\ \mathrm{Au}-3.51077929891264 & 1.34954454527184 & 0.52803892919392 \\ \mathrm{Au}-4.38494212087609 & 4.05453112650669 & 0.85162873551470 \\ \mathrm{Au}-3.33597660227352 & 2.09901304424187 & -4.09918569252215 \\ \mathrm{Au}-3.93211152414376 & 3.14467859975229 & -1.63938617279960 \\ \mathrm{Au}-3.84949404331975 & 2.28081731118764 & 3.22298121946450 \\ \mathrm{Au}-1.87655757107413 & -2.73307242657942 & 2.34966131681934 \\ \mathrm{Au}-2.92836902326811 & -0.21120486614094 & 2.82911387827054 \\ \mathrm{Au}-4.69237869336001 & 4.88647267494731 & 3.45827623186399 \\ \mathrm{Au}-6.60171091355132 & -16.51282973124352 & 5.34221501998191\end{array}$




$\begin{array}{llll}\mathrm{Au} & -5.22328673332511 & -14.14747934091046 & 5.09686642400412 \\ \mathrm{Au}-5.08715948682899 & -15.87971330933288 & 7.55337209841477 \\ \mathrm{Au}-3.90112672085508 & -16.84373960817080 & 4.94487449109497 \\ \mathrm{Au}-2.41170110848369 & -14.42193176908406 & 4.68256315002264 \\ \mathrm{Au}-2.27999150078268 & -16.17551968642146 & 7.19269911793909 \\ \mathrm{Au}-1.23073032029436 & -17.01828899419512 & 4.67719900601350 \\ \mathrm{Au}-3.73591940378209 & -11.91538813397442 & 4.96898212882665 \\ \mathrm{Au}-3.47518883674577 & -15.19562005884925 & 9.59609049446639 \\ \mathrm{Au}-3.62248365593546 & -13.42782601633831 & 7.37045313332667 \\ \mathrm{Au}-1.94164073449273 & -11.08332299335843 & 7.16251286585429 \\ \mathrm{Au}-1.81976644399788 & -12.63370122389402 & 9.39869811758023 \\ \mathrm{Au}-0.57307808117482 & -13.62695592755012 & 7.06320227296593 \\ \mathrm{Au} & 0.43754930084013 & -14.51558757700611 & 4.52596638927771 \\ \mathrm{Au}-2.08541741792254 & -9.69224605148966 & 4.85690645700493 \\ \mathrm{Au}-0.74205247929116 & -12.10769489410767 & 4.66600861710556 \\ \mathrm{Au} & 0.55915868958412 & -16.25257325680797 & 6.98322669375202 \\ \mathrm{Au}-1.72136861647993 & -14.40265051367320 & 11.54455603388865 \\ \mathrm{Au}-0.51134483655313 & -15.39259431667338 & 9.29612175049773 \\ \mathrm{Au} & 1.51458400715855 & -17.04447917440206 & 4.52474057666884 \\ \mathrm{C} & -1.11955897239888 & -6.05719955254396 & 3.30726811140400 \\ \mathrm{H} & -2.10726023093969 & -6.33702049162061 & 3.74658654138863 \\ \mathrm{C}-0.48518167902924 & -5.00370118526668 & 4.21286303798443 \\ \mathrm{H} & -0.25352287624128 & -5.44587835609453 & 5.21149754827802 \\ \mathrm{H} & 0.47102371360413 & -4.64370452681889 & 3.77542686365280 \\ \mathrm{H} & -1.32941110102112 & -5.58886338015019 & 2.31397388750007 \\ \mathrm{~N}-1.37988628779259 & -3.83265399893699 & 4.34223252089517 \\ \mathrm{H} & -2.26042678639203 & -4.08797107187541 & 4.81819619763930 \\ \mathrm{H} & -0.94287304242234 & -3.08769477079639 & 4.90730237601696 \\ \mathrm{~N} & -2.20310646941113 & -8.79418974050769 & 2.70602789361820 \\ \mathrm{H} & -2.91119694124333 & -8.04748003012302 & 2.63095290841869 \\ \mathrm{H} & -2.55789028530897 & -9.59279594308496 & 2.15712797763208 \\ \mathrm{C}-0.88299928744980 & -8.34945740574985 & 2.20023269533124 \\ \mathrm{H}-0.23384216985460 & -9.24918264603766 & 2.15033401871978 \\ \mathrm{H} & -0.97310213319085 & -7.93773738038080 & 1.16617325315740 \\ \mathrm{C}-0.25478179612827 & -7.31098563098009 & 3.13164092748932 \\ \mathrm{H}-0.07607403918053 & -7.79071266307277 & 4.12500542004797 \\ \mathrm{H} & 0.74230175417238 & -7.03024016750977 & 2.72345290433751\end{array}$

Table SC7. Equilibrium geometry for the G11-9 C4-Diamine conformation.
Au 3.03312823265537
Au 0.62194511027622
4.07055582891249
$-0.50656832930020$
Au 1.54306177493068
Au 0.87092825206162
$\mathrm{Au}-1.65943408219636$
Au -0.71641262359714
$\mathrm{Au}-1.34470881253950$
3.79264369678638
$-1.80728798317517$
1.93613572434512
0.37848315744239
4.79938914780980
1.02756333699296
4.50154851107196
2.62835368494535
$-0.25682752023482$
1.97888363764085
5.37471556400257
3.46927004787248
Au -1.80718256043894
$\mathrm{Au}-0.07577677678532$
Au -0.98411273010996
Au -3.59394805644222
Au - 2.75528369295046
$\mathrm{Au}-3.51237131634876$
$\mathrm{Au}-4.06651633279136$
$-0.04195256682643$
1.58988423486323
1.18917798140974
$-0.47022795289421$
2.24428910085177
5.00580866443048
$\mathrm{Au}-4.33816499411601$
3.05461951150404
2.43967475061486
$-2.91694339316106$
1.20387926081809
$-0.94184363231418$
$-1.95380511218152$
0.03387305984615
0.66820321646443
1.17672691292707
$-3.87876951780194$ 
Au -4.26967618154224

Au -3.15435900225260

$\mathrm{Au}-1.85397813192051$

$\mathrm{Au}-2.55642808331406$

Au -3.70294641937015

Au -5.93985522395876

$\mathrm{Au}-4.76623628838332$

Au -5.12743131423040

$\mathrm{Au}-3.22151122981902$

$\mathrm{Au}-1.94478537953728$

Au -2.30257093829192

Au -0.56606520239535

$\mathrm{Au}-3.46243324001278$

$\mathrm{Au}-4.18336989589857$

$\mathrm{Au}-3.92282813970386$

$\mathrm{Au}-2.46148022272962$

Au -2.76809024462831

Au -0.84242163824209

Au 0.84832647694844

Au -2.10799686311234

Au -0.57801944949981

Au 0.49514098428974

Au -3.07148542382434

$\mathrm{Au}-1.21284845977181$

Au 2.11626725083469

C -0.90757994558801

H -1.60676030907708

C -0.06482082565761

H 0.55977477629824

H 0.63190428547326

H -1.54453770562294

N -0.91156159867814

H -1.63951714238266

H -0.34748954057281

N -1.46314852655526

H -0.80090631464313

H -2.27342119207412

C -0.81473408176755

H -0.12239776607543

H -1.60857130948708

C -0.04251817130970

H 0.74416230716730

H 0.50614938568218
4.10455457738426

3.15888204919029

$-1.98072950456874$

0.60687394619571

5.81992884677770

$-17.67541252524617$

$-15.16439064431189$

$-16.93837110923060$

$-17.77220448789949$

$-15.22979228454136$

$-16.92786262047040$

$-17.69536505241798$

$-12.80321000360825$

$-16.11003161691810$

$-14.36443128688328$

$-11.92691824937463$

$-13.43649956885148$

$-14.26217095226749$

$-15.02244794857853$

$-10.44458938036015$

$-12.75080377058029$

$-16.76446383978838$

$-15.17112780818201$

$-16.01494385393871$

$-17.44643925274736$

$-5.40234943394811$

$-5.53184638615029$

$-4.15309599442167$

$-4.30905561723710$

$-3.97222436250187$

$-5.24307777879031$

$-2.94992108523331$

$-3.08936968103870$

$-2.14119637450325$

$-8.26369625893098$

$-8.09107420150757$

$-7.64577279186022$

$-7.98758824170490$

$-8.83001643942099$

$-8.02369292995398$

$-6.66094266434369$

$-6.68623085797827$

$-6.60853224544747$
$-1.34989848502796$

3.36839815050912

2.07860785623040

2.78548163401155

3.78290594073886

4.54737396347016

4.60981030193820

7.06094945662537

4.89789657738615

4.96047799355512

7.46375259003406

5.33722838559303

4.84797256940745

9.44333532373564

7.20561016545151

7.44479227863141

9.65425204302124

7.71805012435437

5.54890502494251

5.12796006169821

5.31066545204665

7.99686373843406

11.77584110329776

9.93632273678412

5.88580752779274

3.58981181248024

4.45188606854499

3.84667052002548

4.75982921975451

2.99921632895501

2.68847756761343

3.99004080655807

4.70961815630695

4.29687055096217

4.77333717104241

5.54771590455559

4.93353450105137

3.46543359547847

3.25535577735918

2.68868506241071

3.41887100864837

4.21154441177640

2.45109091405773

Table SC8. Equilibrium geometry for the A11-1 C4-Diamine conformation.

$\begin{array}{cc}\text { Au } & 3.04715951908938 \\ \mathrm{Au} & 0.93478486871197 \\ \mathrm{Au} & 1.55696688250834 \\ \mathrm{Au} & 0.61164507809651 \\ \mathrm{Au} & -1.63967879950097 \\ \mathrm{Au} & -0.96731988183680 \\ \mathrm{Au} & -1.84892236107216 \\ \mathrm{Au} & -1.23435576537014 \\ \mathrm{Au} & -0.10678433920702\end{array}$
5.03289397321082 4.62905081970526

2.78259340216501

5.64985791274666

5.15984675678751

3.39005592666470

6.08557390012783

4.14820574001750

0.71746010046407
0.09395358954588

$-1.60661210116604$

0.73987285680050

1.19102440899159

$-0.49400199048884$

1.89169019972592

2.19293489527483

$-3.12641696171808$

1.30146653799763 
$\mathrm{Au}-0.63325093891413$

$\mathrm{Au}-3.03391087826212$

$\mathrm{Au}-2.44324151281595$

Au -3.49348757665762

$\mathrm{Au}-4.28473927746714$

Au -3.54136031025991

Au -3.99486820331706

Au -3.64729162400030

$\mathrm{Au}-1.83422279860306$

$\mathrm{Au}-2.76156032439422$

$\mathrm{Au}-4.42235332878702$

Au -6.84861090646289

$\mathrm{Au}-5.40708368496990$

$\mathrm{Au}-5.07211119126137$

$\mathrm{Au}-4.23305383556857$

$\mathrm{Au}-2.69546267157328$

$\mathrm{Au}-2.32232520409723$

$\mathrm{Au}-1.61917361601124$

Au -3.80052727310270

Au -3.21396975538239

$\mathrm{Au}-3.56620807924299$

$\mathrm{Au}-1.80918238518501$

Au -1.47614705284292

$\mathrm{Au}-0.53120868201827$

$\mathrm{Au} 0.12064178478002$

Au -2.12775120244648

Au -0.96819602548097

Au 0.46905330670758

Au -1.20630043137239

Au -0.28609354416621

Au 1.08544448636294

C -2.48216433661780

H -3.27128959285836

C -1.81116772645921

H -1.29658957988542

H -1.03110193634109

H -3.00462881965775

N -2.75049760926943

H -3.24074730636784

H -3.48347554500005

N -1.21340089444347

H -0.47488877228016

H $\quad-0.73025971337397$

C -2.15289196843181

H -2.66504332753164

H -2.93473820888370

C -1.48275093026893

H -0.96267936539711

H $\quad-0.69179618370252$
2.33189457310729

1.80182401406434

0.22384559367897

2.82414315544038

5.54081296676894

3.58912337649332

4.62611944218329

3.73760568924090

$-1.32243040119179$

1.23454876797594

6.36674038308386

$-17.73801570301641$

$-15.40182542370694$

$-17.21323907064362$

$-18.20392426553618$

$-15.85239177559528$

$-17.58615972865468$

$-18.49236487167111$

$-13.23810235376504$

$-16.60351281862576$

$-14.82449644477656$

$-12.60708074242000$

$-14.14668586553926$

$-15.14043318124279$

$-16.04427970010821$

$-11.10288862641522$

$-13.59874490486444$

$-17.80561081490403$

$-15.90296933249257$

$-16.91105434122355$

$-18.62160114515142$

$-5.68967756876320$

$-5.49821478493527$

$-4.36354083922911$

$-3.91833642362562$

$-4.52766636153230$

$-6.10766409997630$

$-3.34656903298162$

$-3.70016700473047$

$-3.13190102310948$

$-9.06978913817779$

$-9.27519090531584$

$-8.72258324712288$

$-8.05144910582299$

$-8.49300095852743$

$-7.89209172610628$

$-6.72303311412592$

$-6.30698171529424$

$-6.91141382092835$
$-1.01129302269719$

$-2.48114706781640$

$-0.38249877442633$

0.14295606126065

0.50328189183124

$-4.51504693110807$

$-2.01081296259349$

2.84149543508703

1.84143306539815

2.39754334839764

3.12078896366197

6.35011356448341

5.93755406914380

8.37205405743714

5.67125023760867

5.22224870252671

7.70092698058973

5.09242950744759

5.64525648852820

10.22383253375751

8.02345183036322

7.67623248096167

9.86006203412050

7.35932480726844

4.73099086080746

5.36513494281235

5.00010774026037

7.15852618414101

11.96751086913722

9.58214139728737

4.60672197653022

3.35190177240185

4.11745379245533

2.97176617345912

3.85083881065451

2.19694331742330

2.45868152579075

2.44636684433640

1.60858635867790

3.14197712622877

4.78791653965723

4.09530240402573

5.63242671230585

4.26543421062806

3.38309135304469

5.03943202524796

3.89177539398033

4.78725075933499

3.12730085291467

Table SC9. Equilibrium geometry for the A11-2 C4-Diamine conformation.

Au 1.40624760514156

$\mathrm{Au}-1.21234724753690$

Au 0.41529147529662
3.28645549361238

3.74236499066098

1.43612178278262
$-3.64655760302311$

$-2.98357568192989$

$-1.81999442176986$ 
Au 1.06143759513765

Au -1.65096963593572

Au 0.04966426354668

Au 0.58378395476409

Au -3.74730136889517

Au -0.67056459814791

Au -2.28575435541564

Au -4.94394088119904

Au -3.47028780453561

Au -2.87135885968906

Au -2.25393965880597

Au -6.28644418124620

Au -4.30342582851936

Au -0.60167079502946

Au -1.84551363876495

Au -1.17136470891885

Au -0.05938522395116

Au -4.12442208464084

Au -3.50887279986323

Au -4.62724322642602

Au -1.86357248655755

Au -1.16873368156554

Au -2.31103535957229

Au 0.27757609839050

$\mathrm{Au}-2.83704464653490$

Au -4.97548617553556

Au -4.03034901168446

Au -3.24059633328775

Au -4.24212864273347

Au -1.55796899068167

Au 1.01513405134588

Au -2.11646136537284

Au -0.48329948459442

Au -0.10388721291500

Au -5.17451329455788

Au -2.58669927125032

Au 2.36684068250811

C -2.47357494246038

H -3.50900139030563

C -2.53019452889372

H -3.13297004031595

H -1.51055588728493

H -1.89170449568883

N -3.09582954649495

H -4.00157986055447

H -3.28604850357442

N -1.53375715585840

H -0.50168242192465

H -1.81661876201879

C -2.10601655463716

H -1.67453288167595

H -3.19701618651357

C -1.86493698682337

H -2.30646872728755

H -0.77027733669330
4.34391772551126

4.78367451880526

2.49797772629859

5.25009034005633

4.10998135092864

$-0.20661028378218$

1.88235945190435

2.28220242664732

0.27694585365126

2.93132912782206

5.66593766775053

4.43957186829827

5.12928276571950

3.41562225086507

$-1.69860153284452$

0.87319306271231

6.06890526037889

$-18.45995752259332$

$-15.78787277602453$

$-17.66673811292595$

$-18.12175529627678$

$-15.40652110943724$

$-17.20998840027310$

$-17.62731206182438$

$-13.24411722585312$

$-16.75430491390673$

$-14.93512674152186$

$-12.29752327987026$

$-13.89306064034532$

$-14.34206246791226$

$-14.77862897203558$

$-10.72671658516180$

$-12.75993582160388$

$-16.59981889121450$

$-15.70372272468113$

$-16.18489690704769$

$-16.96757007869829$

$-5.00606359118962$

$-5.31624562311378$

$-3.62057479759495$

$-3.67609849456787$

$-3.27988027993719$

$-4.95234732311046$

$-2.61335373816779$

$-2.93020058781965$

$-1.72942172208054$

$-8.50096233762378$

$-8.46454772087216$

$-8.29007197240913$

$-7.49940474093949$

$-7.68966623290159$

$-7.69905198955453$

$-6.05198949820057$

$-5.92543083204232$

$-5.86853722611448$
$-1.14318606034377$

$-0.35847563763014$

0.77469189313288

1.34404097207743

$-2.16252368468426$

0.06089936813558

$-1.11388985100624$

$-0.09876391929803$

0.90875801775708

1.75629298561153

2.28707725873416

$-1.14633798371197$

0.62949060442414

3.41725286978324

1.97695938117578

2.76391312786705

3.89354227774203

3.38535050852955

3.82995327719719

5.96021168196020

4.89720369037439

5.38909583434012

7.56540059364425

6.45195803413769

4.49653797540890

8.46935939581660

6.48703931634864

7.22434941282582

9.14744207908103

8.29634178999588

7.13751568585349

5.22960866641327

6.18287744725175

9.26418562180084

11.00351203307408

10.21411885121293

8.11650857593967

4.01496504201426

3.73500892019304

4.65958535066064

5.59858926414702

4.94368307829050

3.06568748322256

3.73708646575641

3.35396446529191

4.23619243433190

5.44353528150737

5.43335003044671

6.41498151004216

4.51333684803995

3.50702395653964

4.43521595778217

4.96242289551004

5.98040728480142

5.07201537258680 
Table SC10. Equilibrium geometry for the A11-3 C4-Diamine conformation.

\begin{tabular}{|c|c|c|c|}
\hline $\mathrm{Au}$ & & 31561696668565 & -2.06830320122522 \\
\hline $\mathrm{Au}$ & -0.18017770460730 & 3.37321364889561 & -2.43606487894929 \\
\hline $\mathrm{Au}$ & 1.20238121118134 & 1.36022674551572 & -0.67639909518534 \\
\hline $\mathrm{Au}$ & 1.16493279192217 & 4.26486903985136 & 0.11763325464436 \\
\hline $\mathrm{Au}$ & -1.66508079598100 & 4.31346051255615 & -0.18906589672445 \\
\hline $\mathrm{Au}$ & -0.25260831575228 & 2.28415874734634 & 1.62007435458063 \\
\hline $\mathrm{Au}$ & -0.31622038141017 & 5.06757135242078 & 2.21494146968977 \\
\hline $\mathrm{Au}$ & -2.86486527392750 & 3.39150361259908 & -2.60608464673259 \\
\hline $\mathrm{Au}$ & -0.24471201583144 & -0.41453765724853 & 0.72875263064898 \\
\hline $\mathrm{Au}$ & -1.63124729312336 & & 023421 \\
\hline $\mathrm{Au}$ & -4.4862005 & 5539 & 745915 \\
\hline $\mathrm{Au}$ & -3.24964704 & 872876 & 5308 \\
\hline $\mathrm{Au}$ & -3.31799269112341 & 2.30407108544362 & 1.42606549291912 \\
\hline $\mathrm{Au}$ & -3.29479091029082 & 5.08670266500265 & 2.02776298277264 \\
\hline $\mathrm{Au}$ & -5.61452529753450 & 3.37268971015989 & -2.58568861945271 \\
\hline $\mathrm{Au}$ & -4.51122186624404 & 4.30296583252392 & -0.24001427749488 \\
\hline $\mathrm{Au}$ & -1.92374518750166 & & 25916 \\
\hline $\mathrm{Au}$ & -1.8 & -2.1 & 6233 \\
\hline $\mathrm{Au}$ & -1.8 & 0.3 & 3592 \\
\hline $\mathrm{Au}$ & -1.939 & 5.72 & 3855 \\
\hline $\mathrm{Au}$ & -6.25944035572744 & -17.31036646447672 & 4.87016809994747 \\
\hline $\mathrm{Au}$ & -4.99206608409008 & -14.83410875921156 & 4.80438478314042 \\
\hline $\mathrm{Au}$ & -5.15000257471506 & 64647 & 7.27849681892581 \\
\hline $\mathrm{Au}$ & & 4838 & 57669 \\
\hline $\mathrm{Au}$ & -2.17207 & 3987 & 21842 \\
\hline $\mathrm{Au}$ & -2.2 & 36 & 7726 \\
\hline $\mathrm{Au}$ & -0.8 & 7643 & 7124 \\
\hline $\mathrm{Au}$ & -3.6 & 3283 & 4503 \\
\hline $\mathrm{Au}$ & -3.93220269582154 & -15.84192239375562 & 9.54653385755246 \\
\hline $\mathrm{Au}$ & -3.838 & 502859 & 7.27996347303610 \\
\hline $\mathrm{Au}$ & -2.29 & 17753 & 03022 \\
\hline $\mathrm{Au}$ & -2.4 & 168 & 2632 \\
\hline $\mathrm{Au}$ & & 391 & \\
\hline $\mathrm{Au}$ & 0.68 & $-14 . c$ & 324 \\
\hline $\mathrm{Au}$ & -2.10 & -10.2 & 79624 \\
\hline $\mathrm{Au}$ & -0.64876540946486 & 92700 & 0132967459 \\
\hline $\mathrm{Au}$ & 0.54525535796382 & -16.65539362072576 & 7.61717743400976 \\
\hline $\mathrm{Au}$ & -2.54461901782823 & 397745910 & 3056279 \\
\hline $\mathrm{Au}$ & -0.91604523314616 & -15.85449671727277 & 78469 \\
\hline $\mathrm{Au}$ & 1.89 & $-17.4 C$ & 8596 \\
\hline C & -1.3 & & \\
\hline $\mathrm{H}$ & & & \\
\hline C & -2.127778099492 & & 886760 \\
\hline $\mathrm{H}$ & -1.89177918975444 & -6.36444476047834 & 1.02058777527742 \\
\hline $\mathrm{H}$ & -3.21972304593845 & -5.53913175445565 & 1.89596038443892 \\
\hline $\mathrm{H}$ & -1.76213402091347 & -4.91177294994308 & 3.74410311402081 \\
\hline $\mathrm{N}$ & -1.83506909345731 & -4.22118056369858 & 1.02097565918076 \\
\hline $\mathrm{H}$ & -0.88434082133277 & -4.22921939881239 & 0.61717005804870 \\
\hline $\mathrm{H}$ & -2.48164 & & \\
\hline $\mathrm{N}$ & & -8.55904508269996 & 5.34909010097886 \\
\hline $\mathrm{H}$ & 0.29230656084685 & -8.93789899378223 & 4.83032835994031 \\
\hline $\mathrm{H}$ & -0.27922403210932 & -8.63637208222918 & 6.35061244582846 \\
\hline
\end{tabular}



C -0.77255769956736
H -1.48070332645549
H 0.16714503111323
C -1.36516521979087
H $\quad-0.78954788615057$
H -2.40433479855755
$-7.15387452544479$
$-6.72583817329150$
$-6.55257116575956$
$-7.06386436482724$
$-7.73098524475922$
$-7.46951742678661$
4.96661971548328
5.70799877987842
5.02587073671067
3.55905599679679
2.87455727317036
3.57944702600272

Table SC11. Equilibrium geometry for the A11-4 C4-Diamine conformation.

\begin{tabular}{|c|c|c|c|}
\hline & & 70884315971023 & -3.90350194934045 \\
\hline $\mathrm{Au}$ & -1.71566225232544 & 3.27247053929029 & -2.84442661210505 \\
\hline $\mathrm{Au}$ & -0.03563751330433 & 0.93593054923946 & -1.95658350642066 \\
\hline $\mathrm{Au}$ & 6871933275231 & 3.74312432190206 & -1.35410888696189 \\
\hline $\mathrm{Au}$ & 6508080072057 & 4.31346043062474 & -0.18906586946593 \\
\hline $\mathrm{Au}$ & 7274027551 & 1.95372505813968 & 95207518 \\
\hline $\mathrm{Au}$ & 0.82233585365846 & 4.65830642080132 & 7453957426175 \\
\hline $\mathrm{Au}$ & 4836 & 3046 & \\
\hline $\mathrm{Au}$ & -0.88167515147713 & -0.63944582749863 & \\
\hline $\mathrm{Au}$ & -2.59812566644109 & 1.46781727351328 & -0.81852276903038 \\
\hline $\mathrm{Au}$ & -5.01283042719901 & 2.01103485559997 & 0.60746748491620 \\
\hline $\mathrm{Au}$ & -3.51384831220632 & -0.07204071684027 & 1.39769408054231 \\
\hline $\mathrm{Au}$ & -2.61882039655884 & 2.52765240007407 & 895308504129 \\
\hline $\mathrm{Au}$ & -1.78150108898661 & 5.21591636288872 & 042667631879 \\
\hline $\mathrm{Au}$ & & & 673 \\
\hline $\mathrm{Au}$ & -4.093918690 & 4.80 & 5576 \\
\hline $\mathrm{Au}$ & -0.12729489787243 & 2.80 & 6575 \\
\hline $\mathrm{Au}$ & -1.85962379421862 & -2.16881650014900 & 36910681026 \\
\hline $\mathrm{Au}$ & -0.96182074463591 & 0.27941079622522 & 2.84546542438092 \\
\hline $\mathrm{Au}$ & 0.63454687879725 & 5.45774067114802 & 3.78753633393084 \\
\hline $\mathrm{Au}$ & -4.58086305105255 & 27578306 & 572377 \\
\hline $\mathrm{Au}$ & 07 & & 3799 \\
\hline $\mathrm{Au}$ & & -17 & 5334 \\
\hline $\mathrm{Au}$ & -2.16 & 9900557 & 97192 \\
\hline $\mathrm{Au}$ & -1.33718449047447 & 185548127452 & 068483965011 \\
\hline $\mathrm{Au}$ & -2.29692419753209 & -16.73977339222595 & 7.39599085778414 \\
\hline $\mathrm{Au}$ & 0.13806893069086 & -17.23186344828399 & 6.00677245179531 \\
\hline $\mathrm{Au}$ & -2.99547298443503 & -12.70569205840542 & 7807679665 \\
\hline $\mathrm{Au}$ & -4.83 & -16 . & \\
\hline $\mathrm{Au}$ & -4.0 & & \\
\hline u & & -11 & 7314 \\
\hline $\mathrm{Au}$ & 537 & -13.3 & 273574 \\
\hline $\mathrm{Au}$ & -1.37016956708441 & -13.89214975512072 & 8.02847136540132 \\
\hline $\mathrm{Au}$ & 1.05087216690289 & -14.43202044095533 & 6.61135242905329 \\
\hline $\mathrm{Au}$ & -2.10235020702960 & -10.25650353786889 & 5.06019890318291 \\
\hline $\mathrm{Au}$ & -0.44838450794076 & -12.35075735343919 & 5.81571701017330 \\
\hline $\mathrm{Au}$ & 0.09620841164963 & -16.20035 & \\
\hline $\mathrm{Au}$ & & & 28087 \\
\hline $\mathrm{Au}$ & -2.25759748052907 & -15.6 & 0784969 \\
\hline $\mathrm{Au}$ & 2.41617010022624 & -16.667205 & 7.44866543734898 \\
\hline C & -2.27209502149401 & -5.55503198335042 & 3.33698319598646 \\
\hline $\mathrm{H}$ & -1.73677455210382 & -4.68336314329148 & 3.78488242720735 \\
\hline C & -2.18567052007256 & -5.44608568584632 & 1.81536931426358 \\
\hline $\mathrm{H}$ & -1.12343856992898 & -5.47229709882736 & 1.48905810773314 \\
\hline $\mathrm{H}$ & .70135345985351 & -6.31444892168844 & 1.33867094391849 \\
\hline $\mathrm{H}$ & 6167829428 & -5.463371 & \\
\hline
\end{tabular}


$\begin{array}{cc}\mathrm{N} & -2.75926018422147 \\ \mathrm{H} & -2.66112376112099 \\ \mathrm{H} & -3.77075095558356 \\ \mathrm{~N} & -1.20174853650853 \\ \mathrm{H} & -0.19047789935599 \\ \mathrm{H} & -1.29854650061960 \\ \mathrm{C} & -1.77578842826637 \\ \mathrm{H} & -2.83789237907602 \\ \mathrm{H} & -1.26002472952512 \\ \mathrm{C} & -1.68978460694253 \\ \mathrm{H} & -0.62368308909948 \\ \mathrm{H} & -2.22523133777716\end{array}$
$-4.16674228168962$ $-4.06379465015546$ $-4.11774991086582$ $-8.25729947149032$ $-8.30636353704585$ $-8.35927083854116$ $-6.97821866222283$ $-6.95205603280825$ $-6.10964105970560$ $-6.86964193690652$ $-6.96137891276511$ $-7.74133401374890$
1.34772685764022

0.32544771248638

1.55191113887351

5.85611163214811

5.65081882364911

6.87862288831670

5.38822957397272

5.71494492143860

5.86445477544815

3.86657311791217

3.54961737783901

3.41889227979403

Table SC12. Lowest-energy geometry from PES of the G12-2 C4-Diamine conformation.

Au 2.87301554438653

Au 1.02536918710864

Au 1.41214203481034

Au 0.28013057648073

Au -1.69329382992937

Au -1.27566105923055

Au -2.31099597442964

$\mathrm{Au}-0.88416421684797$

$\mathrm{Au}-0.16634601635934$

$\mathrm{Au}-0.56016674185666$

$\mathrm{Au}-2.64147667490573$

$\mathrm{Au}-2.31053884485637$

$\mathrm{Au}-3.41849014046533$

Au -4.46764329638047

Au -2.94465053259164

$\mathrm{Au}-3.78710670178839$

$\mathrm{Au}-4.06879308864757$

Au - 1.88784729436072

Au -3.02304333930605

Au -4.99425901100393

$\mathrm{Au}-1.82774009648558$

Au -1.89516936067741

Au -3.54193600413107

$\mathrm{Au}-0.54534215423436$

$\mathrm{Au}-0.56077253517225$

Au - 2.26870176562053

Au 0.57904808387313

Au -1.97494088911484

Au -5.09648648069814

$\mathrm{Au}-3.67076122319343$

Au -3.66557287798345

Au -5.18351723325971

Au -2.36171067270719

Au 0.48600515084154

Au -2.07412769567265

$\mathrm{Au}-0.77181087208963$

Au -1.15552278952260

Au -6.54647946471933

$\mathrm{Au}-3.83685654628626$

Au 1.58576500108755
3.41025945520156

2.89695173665950

1.10398566017025

3.88995526877613

3.37303460989976

1.55197887792698

4.20390503751503

2.39882183153973

$-1.04206679191365$

0.52751960011336

0.00462842450532

$-1.73570548720456$

0.81271400141703

3.48418069324119

1.82511361607683

2.70432465429313

1.76629233472935

$-3.10924410711291$

$-0.68418548473121$

4.36100591686168

$-17.23924558051983$

$-14.53720131705595$

$-16.58152100890057$

$-16.56745174625920$

$-13.78423668281684$

$-15.79934754048480$

$-15.76888196014392$

$-11.95259733298677$

$-15.79839781668620$

$-13.79863973266717$

$-11.08619569366142$

$-12.87226116861550$

$-12.88539280855692$

$-12.83173455275117$

$-9.31607470777116$

$-11.06175513308938$

$-14.90051875371669$

$-14.85797958310654$

$-14.91393485975619$

$-14.81113367846623$
1.37131430531269

$-0.60354833111287$

1.73730867296526

2.14566297324639

0.14975715521259

2.60227420143501

2.80304965973752

$-2.41496093814358$

2.07099617736418

$-0.25241054840993$

$-2.11738487412557$

$-0.05287802422623$

0.49094270600504

0.79203748664863

$-4.15193759395686$

$-1.67501208577544$

3.14785359148338

2.48518814618205

2.85941113554507

3.34804969511828

2.58302201257924

3.12127554353981

4.62805346277298

4.91997687098072

5.53661219542048

7.05717213812098

7.23200298515307

3.86809162561096

6.68088614533837

5.23343857269691

6.12786081824892

7.46336316850185

7.91897294749455

8.00420591926812

4.72137976575456

6.39192437002141

9.52444851759290

8.81508786358243

9.26682563503524

9.59749832783566 


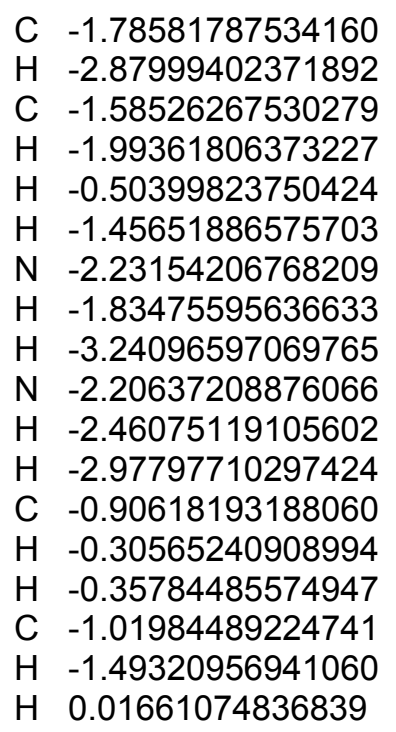

$-6.15564367939768$

$-6.00544542544811$

$-7.43598423346348$

$-8.32357576835958$

$-7.63218201784506$

$-5.28441727167155$

$-7.37062087632463$

$-6.59457852692505$

$-7.17174156800656$

$-3.98096957446041$

$-4.02076181726467$

$-4.41402576072216$

$-4.64727874098084$

$-4.61761266260978$

$-4.03318095827514$

$-6.08470164479131$

$-6.75276432453031$

$-6.46412417277850$
1.34914994972963

1.18551714001496

2.16563009977719

1.63493749485749

2.32989296286023

1.98975138005323

3.49988473120669

4.05594047697134

3.40617000077108

$-0.79110907062382$

$-1.78796926972773$

$-0.26236558067048$

$-0.51729671865485$

$-1.44995013223946$

0.23443930551112

0.01750250392767

$-0.73960308332067$

0.16904028158033

Table SC13. Lowest-energy geometry from PES of the G12-3 C4-Diamine conformation.

\begin{tabular}{|c|c|c|}
\hline $\mathrm{Au} \quad-0.77884368406079$ & 1.58372612892603 & -4.15299459090339 \\
\hline $\mathrm{Au} \quad-2.70167101129220$ & 2.27859327981273 & -2.29488845846249 \\
\hline Au -1.09333925682025 & -0.18954237592267 & -2.07705083697322 \\
\hline Au 0.24610061878124 & 2.47689519126744 & -1.76074634449725 \\
\hline $\mathrm{Au}-1.69471448787574$ & 3.32601162742252 & 0.16669388596501 \\
\hline Au -0.08332057011996 & 0.68469502642211 & 0.44463560564008 \\
\hline Au 1.12691118290093 & 3.27775962557019 & 0.64189383635310 \\
\hline Au - -4.45674662214276 & 2.90534583764443 & -0.38020437066469 \\
\hline-1.37764647713323 & -1.86494169628123 & 0.04003850832067 \\
\hline-3.00629498435344 & 0.43048362766515 & -0.07282212137535 \\
\hline-4.84665177453004 & 1.31677727870412 & 2.03998684217703 \\
\hline-3.43890192640581 & 6721573 & \\
\hline $\mathrm{Au}-2.08162753544272$ & 1.54682408932311 & 6963784548 \\
\hline $\mathrm{Au} \quad-0.83711258858502$ & 4.10476124257095 & 2.77312172454770 \\
\hline $\mathrm{Au}-6.13306663830026$ & 3.73301572805301 & 1.64318831264994 \\
\hline $\mathrm{Au} \quad-3.48101106215184$ & 4.00739319367324 & 2.27586038172842 \\
\hline Au 0.75230768305452 & 1.53686723222114 & 3.03702007866209 \\
\hline$-1.009<5$ & 75132 & 2.50 \\
\hline $\mathrm{Au}$ & 2768 & 953 \\
\hline $\mathrm{Au}$ & 23046 & 3.17 \\
\hline-1.713627638 & 1774285 & 81671 \\
\hline-1.81045344280643 & 84017975 & 6547769 \\
\hline $\mathrm{Au} \quad-3.47878965447113$ & -16.54413689687956 & 4.58425478821962 \\
\hline-0.48862467389860 & -16.50105494955185 & 4.93863482879288 \\
\hline-0.54699441654352 & -13.71629007187340 & 5.549529094279 \\
\hline-2.26729054745696 & -15.75232612397777 & 7.04023130285254 \\
\hline 0.57449279393498 & -15.69246584351099 & 7.27404610077657 \\
\hline-1.94664947344847 & -11.90211425735758 & 3.84593256999374 \\
\hline-5.08644348747548 & -15.76848869360481 & 6.599439526 \\
\hline-3.64478799494404 & -13.76203459116557 & 5.18484318181252 \\
\hline-3.67401182008672 & -11.02726051398913 & 6.04089081289012 \\
\hline-5.21222752814078 & -12.82386515539558 & 7.36181553054475 \\
\hline-2.41792326111653 & -12.82911459202129 & 7.88342754877473 \\
\hline .43939174009193 & & \\
\hline
\end{tabular}


Au -2.07271647583132

Au -0.77518826149205

Au -1.22996228924895

Au -6.59508972314993

Au -3.90247655205662

Au 1.51394122037084

C -0.76442104545095

H -0.64309383961977

C -1.66707583013820

H -2.70602839961601

H -1.72006819751305

H 0.25396790838853

N -1.21446692347538

H -0.20052928739529

H -1.35415035682683

N 0.13212340474719

H 0.60199731890843

H 0.82121841406073

C -0.33126356095656

H -0.81849480710739

H 0.53993700647727

C -1.32478060804006

H -2.22927227258150

H -1.65835894291048
$-9.26905303601569$

$-11.01064837314876$

$-14.83163875966012$

$-14.82965273574545$

$-14.86662761142859$

$-14.73416853513281$

$-6.09733080003634$

$-5.17278284601615$

$-7.08371329110143$

$-6.69389409189603$

$-8.05088247529789$

$-6.54230417626771$

$-7.37010880555740$

$-7.56922305766470$

$-6.54496124132417$

$-3.68006817267226$

$-3.78284866522765$

$-3.25592951004536$

$-4.98271448324532$

$-4.77837521182941$

$-5.65520778606200$

$-5.70348579057455$

$-5.06362236313327$

$-6.62126862604763$
4.70443882220926

6.42721024752175

9.52920457473666

8.68756975419503

9.20423232572881

9.67134343099623

1.49397845365217

2.12625242847762

2.25403994021974

2.31902548206290

1.70806156698969

1.38212721941765

3.63841586988983

3.64937630306907

4.24468121921660

$-0.27225374050730$

0.64126969405397

$-0.91164885423034$

$-0.80206163449089$

$-1.77886694055324$

$-1.00012946803028$

0.11561891621231

0.24878644805616

$-0.42051406538748$

Table SC14. Lowest-energy geometry from PES of the G12-4 C4-Diamine conformation.

$\begin{array}{llcc}\text { Au } & 0.99819889534706 & 1.72151285514847 & -3.31848813883782 \\ \mathrm{Au}-1.56834660785232 & 2.08214326209676 & -2.38759860940530 \\ \mathrm{Au} & 0.13627354812730 & -0.07811961828618 & -1.40721797423524 \\ \mathrm{Au} & 0.92522688799813 & 2.76484805971612 & -0.78445492900641 \\ \mathrm{Au}-1.69471449836633 & 3.32601148512898 & 0.16669394893463 \\ \mathrm{Au}-0.00678642517593 & 0.96461367125674 & 1.23464019573337 \\ \mathrm{Au} & 0.66708437879077 & 3.68140662119951 & 1.73237441371799 \\ \mathrm{Au}-3.98647653351013 & 2.62661681215991 & -1.38877876143856 \\ \mathrm{Au}-0.77354198000082 & -1.67725790048801 & 0.53214064704183 \\ \mathrm{Au}-2.57713857596223 & 0.31209342933090 & -0.34358837744468 \\ \mathrm{Au}-5.11766209818736 & 0.94719639988296 & 0.76296743904871 \\ \mathrm{Au}-3.75157921636917 & -1.18502534583073 & 1.74594294787266 \\ \mathrm{Au}-2.83684518218292 & 1.47034434954022 & 2.38947592810862 \\ \mathrm{Au}-2.06298801072947 & 4.18253787164569 & 2.87682490418283 \\ \mathrm{Au}-6.41949732494969 & 3.16653936349393 & -0.20137790582519 \\ \mathrm{Au}-4.24935223765056 & 3.74879578452753 & 1.38678019416630 \\ \mathrm{Au}-0.45629425750534 & 1.87476192595153 & 3.90355670434544 \\ \mathrm{Au}-1.88925848953499 & -3.15626528413823 & 2.50212887506151 \\ \mathrm{Au}-1.18540186302146 & -0.64444631737935 & 3.31692308429177 \\ \mathrm{Au} & 0.25439803773384 & 4.49725639237774 & 4.32622377208541 \\ \mathrm{Au}-4.26083101639387 & -16.98117475335098 & 2.94050498612707 \\ \mathrm{Au}-3.58991271218720 & -14.33288647862307 & 3.36217370408261 \\ \mathrm{Au}-4.65445778515537 & -16.16213559137385 & 5.53136522918767 \\ \mathrm{Au}-1.93142481883967 & -16.67946493635741 & 4.36419382616472 \\ \mathrm{Au}-1.16374602586817 & -13.97783231238881 & 4.82680900565555 \\ \mathrm{Au}-2.26729055222701 & -15.75232605938718 & 7.04023127947688 \\ \mathrm{Au} 0.27674226708520 & -16.21494492536754 & 5.83628518272538 \\ \mathrm{Au}-2.83095262070924 & -11.81704070422004 & 3.98215201022089\end{array}$


Au -4.88583743944738

Au -3.98721162394485

Au -3.11174055306968

Au -4.07837556174881

Au - 1.43014086758094

Au 1.07891056610373

$\mathrm{Au}-2.07271648068457$

Au -0.45154581243220

Au 0.02058218603486

Au -4.96231077177076

Au -2.41588486568734

Au 2.43627814364505

C -4.34090355277744

H $\quad-5.28109876869284$

C -4.66899329229264

H $\quad-5.36680995826369$

H -3.74099962742545

H -3.72865254383082

N -5.24165258770766

H $\quad-5.93800996496942$

H -5.72847456663751

N -2.45496847569221

H -2.83328919448051

H -1.54063474048978

C -3.36936514830449

H -4.34154875068706

H -2.96107626288273

C -3.57444604512064

H -4.12618547198392

H $\quad-2.58446031421677$
$-15.24450050713250$

$-13.43628849041626$

$-10.81684342880477$

$-12.40268431643315$

$-12.89971715043334$

$-13.34707208438915$

$-9.26905313593090$

$-11.31086679167413$

$-15.17938070344974$

$-14.19819431748082$

$-14.72574697180957$

$-15.57137590338033$

$-4.30628480257158$

$-4.87661111591711$

$-2.83113905731096$

$-2.75695884059370$

$-2.27873710883007$

$-4.37409475830675$

$-2.16269899856113$

$-2.76042636420401$

$-1.29517736547078$

$-6.96948786836164$

$-6.81316328077856$

$-6.48911626283969$

$-6.45145223224878$

$-6.97529536576852$

$-6.79254487574814$

$-4.92771050534207$

$-4.70875823239717$

$-4.42175840878064$
8.05094730540531

6.03509086302059

6.75201763286092

8.69873184891475

7.73880446803460

6.47332866561942

4.70443887896304

5.57048007386754

8.64634279798821

10.59331399686579

9.69671395873708

7.40408496361337

4.30280001017154

4.11006597967699

4.55504213507780

5.42358272916105

4.82882530453683

3.36087378906175

3.36342749456972

2.89140672500312

3.63450567626138

4.38534608879624

3.43768830160059

4.42074305263893

5.43593072509450

5.30706902859961

6.41096417268519

5.48132729716492

6.42526760775286

5.58345618891735

Table SC15. Lowest-energy geometry from PES of the G12-8 C4-Diamine conformation.
Au 2.67923549839590
3.52951347577242
2.92170517660291
1.17052530796934
3.77267637173443
3.32601196020397
1.36296207959752
4.06001576384995
2.26505011636160
$-1.05350546797761$
0.51763246242119
$-0.19989983788226$
$-1.75447729660196$
0.72414411245623
3.38215505538352
1.52970954739168
2.47132677125475
1.68443721402353
$-3.15626542178903$
$-0.83468076517479$
4.24688101636286
$-16.51175611303549$
$-14.00766506147625$
1.85405164607829
$-0.29155355239331$
2.17064597057893
2.41687311060549
0.16669589044452
2.71177348697624
2.77758065144975
$-2.25578853208454$
2.35327419084364
$-0.01756067822912$
$-1.96825060869263$
0.20628394848680
0.53066165433580
0.58195313184510
$-4.10820579839794$
$-1.76096867374242$
3.04494143637073
2.50212896266398
2.99392151875713
3.05776390488603
4.11185353131404
4.18184398575047 
$\mathrm{Au} \quad-5.08994332780758$

$\mathrm{Au}-3.16985690453335$

$\mathrm{Au}-1.89812066140650$

Au -2.26728843672535

Au -0.51698449091276

Au -3.44252967188110

$\mathrm{Au}-4.15659052326251$

Au -3.89238734616917

Au - 2.42918500759858

Au -2.75441283628101

Au -0.81660648212585

Au 0.88530787414354

$\mathrm{Au}-2.07271648117854$

$\mathrm{Au}-0.54502376488205$

Au 0.52518452008432

$\mathrm{Au}-3.06117126724430$

Au -1.19573382383922

Au 2.15922770565669

C -3.35856452652861

H -3.64401470327056

C -1.92770564475182

H -1.24858186601853

H -1.60510539161801

H -4.07155302918882

N -1.75504348583758

H -2.29699480154019

H -0.76716765258185

N -4.90234955403149

H -4.90786826024091

H -5.80708055699233

C -4.75682341481436

H $\quad-4.77664428714737$

H -5.63258277704179

C -3.45662373972498

H -2.60701818421925

H -3.33162519538371
$-15.76715824060848$

$-16.59635225032329$

$-14.04846169174811$

$-15.75232738195917$

$-16.51265883228369$

$-11.64661603228349$

$-14.93621120854281$

$-13.18807216619617$

$-10.74338587591531$

$-12.24785584356578$

$-13.08571276551629$

$-13.83561768082032$

$-9.26905332555650$

$-11.56321021381580$

$-15.58464560305410$

$-13.98916961985016$

$-14.83723644490395$

$-16.25877854849823$

$-5.32526308622554$

$-5.16966180089104$

$-5.87519630696475$

$-5.08333644849453$

$-6.07185513281802$

$-6.07330832817664$

$-7.12950224241448$

$-7.07722484777751$

$-7.21694071967083$

$-2.55229166134913$

$-3.25114748299618$

$-2.05971627664633$

$-3.19725572524828$

$-2.37845766887300$

$-3.85959516608628$

$-3.99233844119749$

$-3.32482535958594$

$-4.19945209433251$
6.63086388085012

4.46954432976546

4.54230863813523

7.04022685103881

4.92175747834558

4.42332505088178

9.01581141398219

6.78460183305035

7.00843174450465

9.22537561425501

7.30905212059721

5.14211770163939

4.70443891815840

4.91210445492041

7.59038290206820

11.34908100173332

9.52125166679179

5.48739058839281

4.53781908626029

3.46617437011256

4.57009967609153

4.15493502070921

5.61521466015855

4.95521581844340

3.78624987720505

2.90776179481942

3.49963519860942

3.80558170781935

3.04550308775087

3.75297674095567

5.13634714861870

5.88593045164017

5.34637320413560

5.31173992438902

5.02192063014754

6.39828497555941

Table SC16. Lowest-energy geometry from PES of the G12-9 C4-Diamine conformation.

Au 2.42583162476875

Au 1.15223360004574

Au 1.11340918428671

$\mathrm{Au}-0.31138169827179$

Au -1.69470601134809

Au -1.74185740152145

Au -3.00124804351684

Au -0.23378745038332

$\mathrm{Au}-0.33037148278108$

$\mathrm{Au}-0.22205858175144$

Au -1.82247797445955

Au -1.86913041973880

$\mathrm{Au}-3.33752258840918$

Au -4.54820632099691

Au -1.78761844425673

Au -3.24919950075024
3.82266634161236

3.13970679281781

1.40580355464966

4.07513154809584

3.32601202556405

1.61031137337902

4.16045426275787

2.39534183864062

$-0.86365220000797$

0.64121243478298

$-0.16663563316361$

$-1.68679652231983$

0.75320580664901

3.32890963996664

1.56350089590608

2.49801638458305
2.42028735843377

0.08243739365123

2.55528678463229

2.54335221022220

0.16669587480573

2.69569368286597

2.56316365107681

$-2.09910869142338$

2.57843102425281

0.15357894679992

$-2.06698600346551$

0.15133719727869

0.18626317787442

0.13477945500714

$-4.20802534400466$

$-2.07196068747042$ 
Au -4.57729742319426

Au -1.88925850431815

Au -3.26497493699282

Au -5.73933111125103

Au -5.72248956788989

Au -4.57320772568942

$\mathrm{Au}-5.06028133280109$

Au -3.01314802074669

$\mathrm{Au}-1.76181686376651$

$\mathrm{Au}-2.26728843674305$

$\mathrm{Au}-0.40064359878803$

Au -3.36679542938186

$\mathrm{Au}-4.25765222851210$

Au -3.91074771272228

Au -2.54173106479646

Au -2.90975936354596

Au -0.87341055572896

Au 0.99693394286507

Au -2.07271648485944

Au -0.31879267005393

Au 0.50311102824879

$\mathrm{Au}-3.27511210135821$

Au -1.29900284642944

Au 2.25379866269885

C 0.56759055342511

H 0.10893951741820

C 1.62046080772170

H 2.45836727413285

H 2.05088039500518

H -0.26780157505811

N 1.05282645211666

H 0.51719887475478

H 1.80430568137876

N -0.86731492553075

H -0.33212939898884

H -1.55595962781799

C 0.03752157715407

H 0.53077443849512

H -0.60576829092483

C $\quad 1.11200650022914$

H 1.82255334423838

H 1.70807507259175
1.55422210235168

$-3.15626551169317$

$-0.82123175464769$

4.07503520111515

$-16.52223882333021$

$-14.02107302630471$

$-15.75712445967813$

$-16.52948799338736$

$-13.95036988810862$

$-15.75232748908232$

$-16.44297366299960$

$-11.63565061140856$

$-14.90776209437656$

$-13.15840070409198$

$-10.67271870978830$

$-12.20287164292664$

$-12.93516773781161$

$-13.80859515417309$

$-9.26905319388928$

$-11.46678931630152$

$-15.43968206912148$

$-13.94841579822263$

$-14.64039322915915$

$-16.21242670268012$

$-7.93355285188975$

$-7.97231351636441$

$-9.03457527719412$

$-8.82498431126515$

$-9.03138776983214$

$-8.16650843390084$

$-10.38155375479345$

$-10.41342445158860$

$-11.08268841196267$

$-5.25545177035938$

$-5.25137786789851$

$-6.02102170129911$

$-5.44261925434916$

$-4.46704370347173$

$-5.66340019972677$

$-6.52935553302833$

$-6.22289825401989$

$-6.55043577090210$
2.60900756081399

2.50212897884290

2.58677811336161

2.48779880083217

3.91287869865477

4.02831889703401

6.46886971969796

4.39219650579981

4.56251043181236

7.04022688855337

4.98444858683937

4.29829896867193

8.89378081078596

6.66793585156938

6.98613522774485

9.15524764950828

7.20308797731550

5.24934168146715

4.70443888010372

4.83580596087734

7.67343963162378

11.26778989294964

9.48120759241506

5.68325897617626

3.29462073600737

2.27773729855982

3.41841908758202

2.70824558029903

4.44345618701782

4.01603695105671

3.18171948537038

2.29974163723308

3.10021199603211

2.59393360853408

1.71018638876232

2.52353592888660

3.75620471534379

3.95496229515916

4.63583804290880

3.58527985262355

2.78043652956412

4.52655409355777

Table SC17. Lowest-energy geometry from PES of A12-2 C4-Diamine conformation.

Au -0.00910912832598

$\mathrm{Au}-2.25355036196664$

Au -0.58509775278321

Au 0.58181071874080

$\mathrm{Au}-1.69471617980211$

Au -0.01768563863232

Au 0.98643721568704

Au -4.32640076747094

$\mathrm{Au}-1.20176781455900$

Au -2.93970617362944
1.56978397401574

2.15996608050094

$-0.18442179705541$

2.56802174601328

3.32601184611185

0.81154839521081

3.45565790732927

2.89994700363757

$-1.72378359048988$

0.44377033547263
$-3.84971035090810$

$-2.36815706712477$

$-1.79124739722187$

$-1.36627238830960$

0.16669276504566

0.80966529773468

1.14083784772206

$-0.84805473993976$

0.30980460178590

$-0.16024283578174$ 
Au -5.08193795499973

$\mathrm{Au}-3.69783878066909$

$\mathrm{Au}-2.45447226034536$

$\mathrm{Au}-1.35605128380603$

$\mathrm{Au}-6.36730046046516$

Au -3.85603747562301

Au 0.23277507666205

$\mathrm{Au}-1.88926017467388$

Au -0.83270692349405

Au 1.25033931650523

Au -2.31165650732409

Au -2.23129451289609

$\mathrm{Au}-3.78909807564629$

Au -0.79328912148801

Au -0.66071729350466

$\mathrm{Au}-2.26728882085933$

Au 0.57872251839021

$\mathrm{Au}-2.15491026683623$

Au -5.11385159392261

Au -3.76023155974328

$\mathrm{Au}-3.61986279531637$

$\mathrm{Au}-5.03013331173860$

Au -2.18292891038928

Au 0.66494662898286

Au - 2.07271482431050

$\mathrm{Au}-0.63806753922336$

$\mathrm{Au}-0.89800318262507$

$\mathrm{Au}-6.32638560796600$

Au -3.58766757768772

Au 1.84404904961627

C -4.27813632269505

H $\quad-5.27465467460605$

C -4.17898851066588

H -4.58196425081305

H -3.11241793583041

H -4.21143490470973

N -4.86632815336035

H $\quad-5.82812888255904$

H -4.93406359795023

N -1.98771103934786

H -1.15603186373741

H -1.79566999871400

C -3.19572334935263

H -3.28933425752596

H -4.07951895059590

C -3.14903012781421

H -3.15963806619974

H -2.17331507392056
1.26707829089643

$-0.99050191469775$

1.56262972714336

4.18674148828394

3.61079294017473

3.97137915075773

1.72316170059282

$-3.15626593004439$

$-0.71188627196460$

4.26508419959805

$-17.17180830505228$

$-14.44700418001614$

$-16.47653360201195$

$-16.56510018343707$

$-13.78940479363340$

$-15.75232493310054$

$-15.81911145772435$

$-11.84714658281528$

$-15.64943924047303$

$-13.69689917196419$

$-10.99132614624122$

$-12.72928242225455$

$-12.81870228632113$

$-12.90321719697197$

$-9.26905298773286$

$-11.08369139872372$

$-14.88415318930778$

$-14.66854718649503$

$-14.80332818918499$

$-14.91531147433181$

$-3.87030705089191$

$-4.29771780302806$

$-2.41602523462658$

$-2.32087139838003$

$-2.09729582717721$

$-3.87335483383580$

$-1.50071561783029$

$-1.81201351232244$

$-0.54884089150426$

$-6.98190617616212$

$-6.59795682018413$

$-6.91241065212674$

$-6.19595819949832$

$-6.21578507657394$

$-6.73276881676740$

$-4.74890521322515$

$-4.73883790568429$

$-4.29297868047304$
1.50312453871801

2.15137089229459

2.57751172528762

2.87952577895802

0.86900467439837

1.92733876960869

3.51024715849468

2.50212767809126

3.11453388295323

3.75815626969524

2.53472339741359

3.07848763424679

4.73629160451438

4.73498549997890

5.34935719189930

7.04023226449121

6.92825436253304

3.85189126897401

6.92926373293676

5.34895563550979

6.23490978192805

7.72946972384067

7.89310625617947

7.73205948692472

4.70444029597502

6.23851398907942

9.38714517086835

9.19561930808791

9.38557852280523

9.19831718645880

4.64163350473741

4.90127696643521

5.11646090679026

6.15351666626396

5.14502378736712

3.52493015813416

4.18185360122184

3.97422085398701

4.57332236469936

5.04209209003539

4.56389814113607

6.05509165481956

4.69079872544367

3.58279909757652

5.09844286602144

5.19911493150008

6.31483729475822

4.88815364125413

Table SC18. Lowest-energy geometry from PES of the A12-3 C4-Diamine conformation.

Au -6.37421955437988

$\mathrm{Au}-3.91856214418378$

$\mathrm{Au}-5.01010448159076$

$\mathrm{Au}-4.26865936007575$

Au -1.69471423318701
3.40470435438792

3.81670056503974

1.11081807944392

2.85812848890186

3.32601193185793
0.71657147197192

1.88771510219815

1.40465381700709

$-0.95877603019880$

0.16669412714104 
$\mathrm{Au} \quad-2.82511199353800$

$\mathrm{Au}-2.10159430765538$

Au -1.46417696501757

Au -3.52777195304787

Au -2.41016969562844

Au 0.21687620741618

Au -0.72038830928209

Au -0.04530065731864

Au 0.66060636948717

Au 1.10758200509993

$\mathrm{Au} 0.94618150246529$

Au -0.43564468201716

Au -1.88925825723303

Au -1.14642056692125

Au 0.20365028668857

Au -0.13360192509249

Au -0.70709148393371

Au 0.17032069521694

Au -2.51852748416729

$\mathrm{Au}-3.17353845267877$

Au -2.26729075703396

Au -4.76689442301111

$\mathrm{Au}-1.33391531844891$

Au 0.32028618063803

$\mathrm{Au}-0.40761511403504$

Au -1.18920466590455

Au -0.38452970796835

Au -2.97322902660230

Au -5.47002646762393

Au -2.07271675748358

Au -3.84948697947140

Au -4.58291219503950

Au 0.33493540424517

Au -2.17739274322477

Au -6.96448000964293

C -1.26215688693018

H -1.68143639701846

C 0.15488295315301

H 0.82710450170427

H 0.55953264027760

H -1.90784172120218

N 0.17393747098036

H -0.28266147667459

H 1.14367448971274

N -3.10750081236534

H -2.99737985244198

H -4.09305231221397

C -2.83520418619366

H -3.06303082700985

H -3.53003148552226

C -1.38759749223234

H -1.05889623836324

H -0.71904136912898
0.48659889382407

2.25844224161398

4.16987487781432

$-1.04405177878684$

1.47247268408723

1.80274554439544

$-0.73359771846526$

0.77592557736566

2.51158745394199

4.39070177722032

3.46984907789008

$-0.16691956310169$

$-3.15626571575594$

$-1.73301058150034$

1.58780785841520

$-14.20304387066433$

$-12.40852289066802$

$-15.24424383510281$

$-14.70499949788365$

$-12.87500663836578$

$-15.75232560525952$

$-15.14533088366342$

$-10.81049883347998$

$-16.16146654187121$

$-13.43428252972878$

$-11.74435538684130$

$-14.29381613612814$

$-13.93502322629786$

$-13.34625397901718$

$-9.26905361575334$

$-11.30527169470431$

$-16.17529490022113$

$-16.96255055793558$

$-16.64915393351468$

$-15.54481052204919$

$-3.92232223688767$

$-3.51735251485971$

$-3.39317285777987$

$-3.75129450785007$

$-3.78857008002888$

$-3.49223131075751$

$-1.91350652712485$

$-1.48355755835525$

$-1.56392598028348$

$-7.29894528082883$

$-7.48424798142666$

$-7.51653224116970$

$-5.87082946696231$

$-5.68708769461410$

$-5.22460181998128$

$-5.45731456137549$

$-5.88192423164657$

$-5.89936804080613$
$-0.32242410845416$

$-2.43855339703390$

2.89544233942616

1.99541332136443

2.59532371290276

3.61982817756529

3.33427660790057

0.97946558338798

$-1.21517025118630$

3.85830391342329

1.26504964055399

$-1.73189114853230$

2.50212938086248

0.32327170595179

$-3.76628325941550$

10.95639449889681

8.95172016108909

8.42515620470690

9.68324379555904

7.59394009959304

7.04023074403095

8.27311845900318

6.88431629871822

5.90006676044994

6.28709293361859

4.08600351040151

3.59122358804771

4.67488796930029

5.96448653756382

4.70443874627690

5.31236631428653

5.43845689668774

3.27895397469452

4.32960088762181

6.66930913360684

5.55290795493903

6.50389211236880

5.33517504579382

6.15285096672965

4.37701820165653

4.73723086512218

5.24040257887376

6.06034026769945

5.22664207295677

5.52783567694275

6.53890144593149

5.30975878303088

5.24168033578286

4.16766101795224

5.83221445285026

5.53383234076477

6.51099511337244

4.75988384124943

Table SC19. Lowest-energy geometry from PES of the A12-4 C4-Diamine conformation. 
$\mathrm{Au}-2.84311871761489$

$\mathrm{Au}-3.75748469405941$

Au - 2.58936263024242

$\mathrm{Au}-0.81976229186008$

$\mathrm{Au}-1.69471538534853$

Au -0.49697337647184

Au 1.04039871915144

Au -4.49477432789032

Au -2.26929858238920

$\mathrm{Au}-3.48355313313293$

$\mathrm{Au}-4.21428567410377$

Au -3.27306883647750

$\mathrm{Au}-1.44334096223716$

Au 0.16883238927208

$\mathrm{Au}-5.13387655182240$

Au - 2.42837625956229

Au 1.34164918043913

Au -1.88925939001242

Au -0.20155339561475

Au 2.79953345603341

Au 1.66232319081984

Au 0.53920034475251

Au -1.08487691064308

Au 0.58124341373797

Au -0.58812017202058

Au -2.26728962460198

Au -0.61640555160192

Au -0.71298389087978

$\mathrm{Au}-3.77025633924656$

Au -2.31496804318248

Au -3.66662046086337

Au -5.14483313344023

Au -3.69770780028215

Au -1.98906174076292

Au -2.07271558909934

$\mathrm{Au}-2.03073097203997$

$\mathrm{Au}-3.60889180710932$

$\mathrm{Au}-6.49016466594541$

$\mathrm{Au}-5.10236356188459$

$\mathrm{Au}-1.96408054134670$

C -4.06715676246191

H $\quad-5.11628633475350$

C -3.74232531816415

H -4.08810313263824

H -2.64124004432797

H -4.02271069267361

N -4.32820523461693

H $\quad-5.32448039098377$

H -4.28634931324044

N -2.00651990974938

H -1.95313087899464

H -1.09730272864457

C -3.10283527038604

H -3.00015058967426

H -4.06499186021584

C -3.08643903985218
1.57219495328146

2.50742230007410

$-0.17522139852106$

2.25144937462644

3.32601165121142

0.48832324847155

2.86072078276874

3.46790603633353

$-1.73201218838569$

0.77052430940341

1.78767281957314

$-0.76120741688098$

1.44393816104724

3.80292763736665

4.37443618050527

4.14507141335586

1.11654830670139

$-3.15626582243512$

$-1.05437511014561$

3.41834965961100

$-16.58133691799011$

$-14.06427451881387$

$-16.61354900158551$

$-15.81318930052333$

$-13.21237835460605$

$-15.75232576775379$

$-14.96256538813726$

$-11.68070520561594$

$-16.48338788107739$

$-14.04109205050274$

$-11.53429847229925$

$-13.78672238892689$

$-13.06279771179749$

$-12.24180779943494$

$-9.26905306064160$

$-10.73488958468585$

$-14.82008813582646$

$-16.18676065179952$

$-15.54046278422413$

$-13.99313181705911$

$-4.36655718465994$

$-4.71022536234455$

$-3.19748777701313$

$-3.42340776611013$

$-3.04449085932776$

$-3.96615756736773$

$-1.92699288173723$

$-2.04273004844069$

$-1.19768507936530$

$-7.42386532703304$

$-7.87180018679943$

$-6.95216969524015$

$-6.42287514599173$

$-5.75303466541359$

$-6.96981360983346$

$-5.55215981772697$
$-4.04597067543316$

$-1.62329457217389$

$-1.92884900275754$

$-2.32035566486750$

0.16669332558542

$-0.10433341554840$

$-0.47134324954932$

0.76120796720802

0.22630971502713

0.66142471883448

3.20841151070915

3.07938019548856

2.67547946739782

2.27234631106110

3.28531689047543

2.81407360474542

1.98627243233299

2.50212848699162

2.28797309108585

1.56072548005835

4.56575615525147

4.49893832629153

4.59246155057890

6.97332510326700

6.98741880627158

7.04023172007823

9.23075964506474

4.58702786485424

4.72853805043844

4.51890228496856

4.73737170270252

4.78521357875103

7.14441678811389

9.26555464340553

4.70443947623965

7.01602171776591

9.38102632663366

4.97640277501472

7.25894106642269

11.41417671582765

4.46964603187788

4.62232803785678

5.40904510419977

6.44606504724280

5.45402632646633

3.42356138888996

4.91365651101651

4.66885718773323

5.64144130264476

3.31259328795098

2.38382358166317

3.45434236323829

3.32144293684581

2.42882703378640

3.22264589015722

4.58175071731375 

H $\quad-3.29359322938546$
$-6.17434021842132$
5.48219191984122
H -2.05234748218559
$-5.14692160166546$
4.70697589009656

\section{Oligophenyl Conformers}

We have considered the binding conformations of biphenyl diamine and limited our investigation to two conformers associated with the relative orientation of the two nitrogen lone pair orbitals. The labeling that will be employed is summarized in Scheme SC3. Also seen in Scheme SC3 are the stacked dimeric junctions that we have studied that included cis-cis (CC11), cis-trans (CT11), and trans-trans (TT11) pairs of biphenyl diamines. The CT11 and CC11 conformers have nearly identical PES, as shown in Figure SC9. It is also noted here that the binding energies for the stacked dimers were calculated as $\Delta E=E-2 \times E\left(A u_{20}\right)-E$ $\left(\mathrm{N}_{2} \mathrm{C}_{12} \mathrm{H}_{12} \cdot \mathrm{N}_{2} \mathrm{C}_{12} \mathrm{H}_{12}\right)$ where the biphenyl diamine was fully optimized. Its' structure is shown in Table SC26 and most resembles the structure at the minimum of TT11.

Scheme SC3. Biphenyl diamine conformers that were hunted for and found along with labeling scheme used.
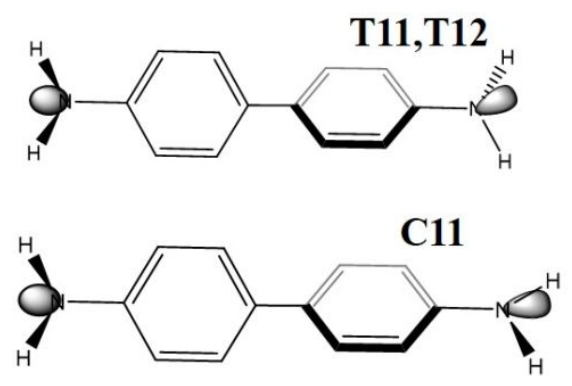

CT11

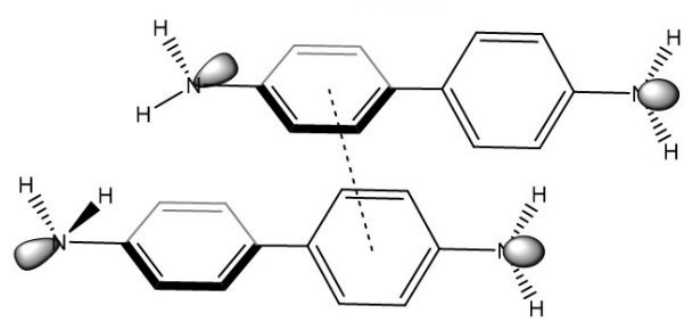

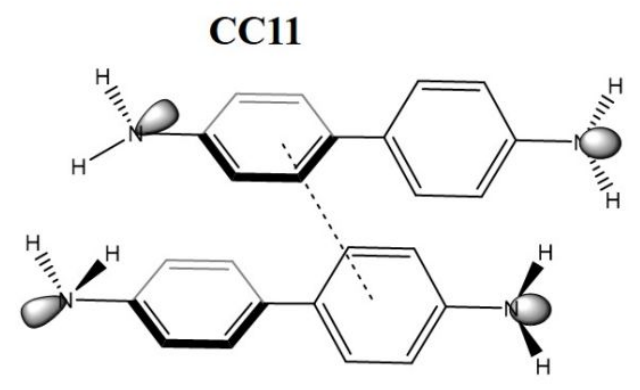

TT11

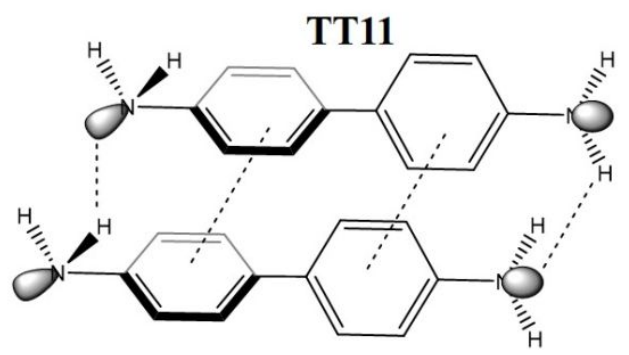




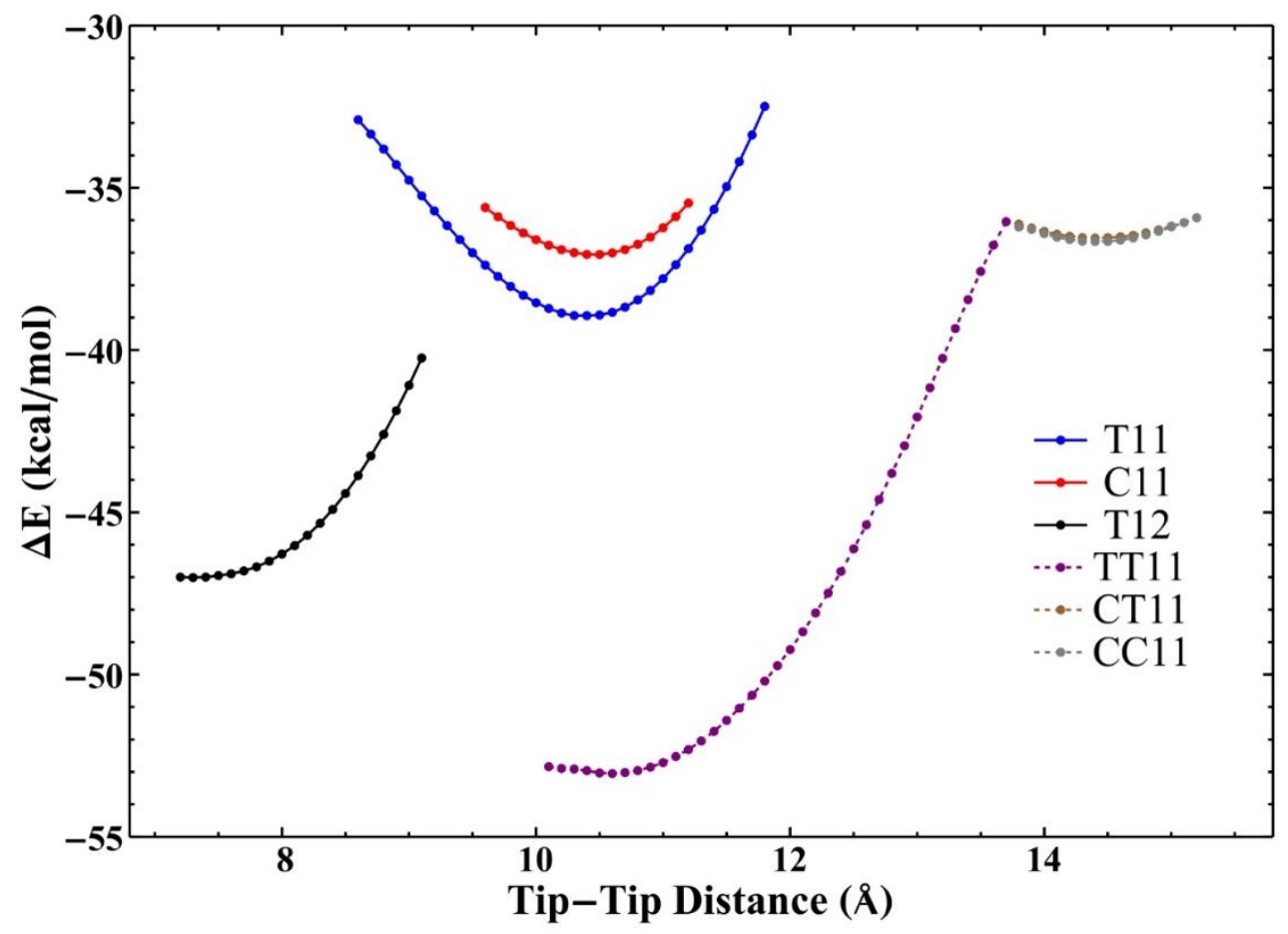

Figure SC9. PES for the all located Biphenyl Diamine conformations calculated at the BP86/DEF2-SV(P)/D3BJ level.

\section{$\mathrm{Ph}_{2}$ Equilibrium Geometries}

Table SC20. Lowest-energy geometry from PES of the T11 Biphenyl Diamine conformation.

Au 3.00480135320657

$\mathrm{Au} 0.45475040384322$

Au 1.53890947066999

Au 1.06229826153153

Au -1.60440055264217

$\mathrm{Au}-0.50333566908781$

$\mathrm{Au}-0.94545383834370$

$\mathrm{Au}-2.10000224888713$

Au -0.04798919827036

$\mathrm{Au}-1.11875218058516$

$\mathrm{Au}-3.87365704892527$

Au -2.90350750455360

Au -3.42054139250128

$\mathrm{Au}-3.78585568389700$

Au -4.75878719219452

Au -4.34173237235672
5.70737974562818

5.53386374110755

3.63541417330584

6.52264450022079

6.33538207502596

4.41873819290494

7.18453668521360

5.31756954381058

1.73220053333233

3.40016463850174

3.17534837790704

1.48941879948163

4.17207148524598

6.94428032480208

5.04904621722547

6.06473365778866
$-1.70795083591840$

$-2.72168436464368$

$-0.64931065120774$

0.06533855422795

$-0.91752470342693$

1.20696717204897

1.72839001644598

$-3.53607865332283$

0.38690121896526

$-1.66390357498169$

$-2.37473707514443$

$-0.52213156522145$

0.27683489521026

0.82233585854068

$-4.18347018935099$

$-1.65811628528184$ 
Au -2.70618324756291

$\mathrm{Au}-1.79894454661918$

$\mathrm{Au}-2.27503193231624$

Au -3.08501508387132

$\mathrm{Au}-0.65399616041728$

$\mathrm{Au}-1.09692200281125$

$\mathrm{Au}-0.07960335965828$

$\mathrm{Au}-2.89049110115152$

Au -3.40595196607898

$\mathrm{Au}-2.35760070753543$

Au -4.98019912527944

Au -1.57990023269838

Au 0.33703884570785

$\mathrm{Au}-0.51131898853169$

$\mathrm{Au}-1.13851180826069$

Au -0.20897137776602

$\mathrm{Au}-2.89995779987860$

$\mathrm{Au}-5.52388069048457$

$\mathrm{Au}-2.16302947258833$

Au -3.92071680867356

$\mathrm{Au}-4.49761454995057$

Au 0.62320646843840

Au -1.98487516408737

$\mathrm{Au}-7.00299231590087$

C -2.01347810227190

C -1.73216121485145

C -2.40621891318443

C -3.36730374297557

C -3.65736286511401

C -2.99053046179969

C -2.12138321563932

C -0.82349949310061

C -0.55051641332641

C -1.57639825855785

C -2.87976804324341

C -3.14131705336114

H -1.48208942611710

H -0.99566644290213

H -3.88580552016036

H -4.40042143145715

N -3.26857727024187

H 0.00303102046459

H 0.47294472216878

N -1.31496326452716

H -3.69605613585651

H $\quad-4.17249449775764$

H -4.25324764353251

H -3.02759294581774

H -0.32300375372948

H $\quad-1.88507742520724$
4.98737851738430

$-0.14689483091533$

2.38912124410963

7.69955059793424

$-17.13731276325144$

$-15.36430999313160$

$-18.17253286048592$

$-17.72045862906385$

$-15.91648212884464$

$-18.76169319343775$

$-18.23580434355940$

$-13.79182748900599$

$-19.08457897299198$

$-16.38421689280622$

$-14.72845981787069$

$-17.24747264896198$

$-16.96765043485461$

$-16.46059620093154$

$-12.27842449436607$

$-14.36625629175065$

$-19.25869167827463$

$-19.88753195837876$

$-19.65272744588409$

$-18.70709269115532$

$-8.16351216242329$

$-7.06182251793791$

$-6.86156565493804$

$-7.82719889966242$

$-8.93167128933502$

$-9.09885301131608$

$-5.68231565351705$

$-5.12437385854130$

$-4.01597932155691$

$-3.43540880426014$

$-3.96401761813410$

$-5.07162956830592$

$-8.28894588110751$

$-6.31891344073449$

$-7.72576145697003$

$-9.67722773643836$

$-10.23775557731118$

$-5.58802776319741$

$-3.60746608437101$

$-2.28387421788774$

$-3.49632231264467$

$-5.45482795093411$

$-10.53660227458745$

$-10.08530888429964$

$-2.19601998018323$

$-2.25614897915779$
2.89279837411415

1.41791026459715

2.22611339353208

3.36239553860423

12.21505409579103

10.15879347938957

9.73031120113393

10.72670019805097

8.57713817439541

8.12445128494379

9.11265237409751

8.03297472217143

7.23259693089625

7.54286228697971

5.27240035660688

4.86048564601610

5.69630999307391

6.74065419652503

5.78865786673153

6.23919599589073

6.31206438502595

4.62826493525340

5.43610164380168

7.31068653709433

5.85250419692218

5.03642470796032

3.80886581060135

3.42494104026572

4.23341271669805

5.46236916220268

2.95770818162926

2.88314104289153

2.07295107094030

1.30341430758995

1.37594347923852

2.18939243804002

6.81090804203893

5.38209085010159

2.45815484660640

3.90417952722957

6.28018217439519

3.44503873041092

2.02531572332775

0.49869658488635

0.80033046769061

2.24964198551405

6.22665398783698

7.27005645051877

0.23551661642959

$-0.35910868873379$

Table SC21. Lowest-energy geometry from PES of the C11 Biphenyl Diamine conformation.

Au 2.64987074805957

Au -0.06716793713264
5.40182112911116

5.44581842199046
$-2.73122735166062$

$-3.14966676283517$ 
Au 1.29591580367308

Au 1.22258115650869

$\mathrm{Au}-1.60298937140943$

Au -0.21190465454487

Au -0.30402965774206

Au -2.74785169964485

$\mathrm{Au}-0.16761331752539$

$\mathrm{Au}-1.53039181894581$

Au -4.38465247983054

$\mathrm{Au}-3.16595871505055$

Au -3.26925271221035

Au -3.28504665684372

$\mathrm{Au}-5.49691011650198$

$\mathrm{Au}-4.44861745775396$

Au -1.92902570818508

$\mathrm{Au}-1.79753336743440$

Au -1.86267398921303

Au -1.97667077158926

$\mathrm{Au}-6.39754281832886$

$\mathrm{Au}-5.10566323138841$

$\mathrm{Au}-3.66534033513697$

Au -5.21089000527474

Au -3.86782870996211

Au -2.35901385453296

Au -3.91748050845040

Au -3.69117560793851

Au -0.97693778870643

Au -2.28149023912678

$\mathrm{Au}-0.72581537903023$

Au 0.58232498424422

Au -0.76319885885038

$\mathrm{Au}-2.36557733622540$

Au -2.16444064925577

$\mathrm{Au}-2.28479724166366$

Au -0.90134231047770

$\mathrm{Au} 1.76812711108764$

Au 0.48919811190612

Au - 2.45727465305003

C -0.16729294707234

C -0.38086303299184

C -1.23672646908864

C -1.90033391140244

C -1.69362813467102

C -0.80345474296909

C -1.39338871887470

C -0.28104696471741

C -0.39441812869465

C -1.63420389920752

C -2.76249620609202

C -2.63816905597588

H 0.50818699364119

H 0.12337503484657

H -2.56417860410619

H -2.20987802756855

N -0.57467854271061

H 0.70634318466665
3.43576559048188

6.34170566200925

6.38240346901513

4.35158061607690

7.13622622764342

5.44476735165997

1.64827268116641

3.42735206696894

3.43659928781863

1.64818541233409

4.34747449223604

7.13198090488075

5.40005657811465

6.33648603179675

5.06457780834249

$-0.09987359020128$

2.45012015410050

7.79125875032196

$-17.67387466530585$

$-15.71806314156908$

$-17.83469664737640$

$-18.66522789071597$

$-16.70655053983927$

$-18.80871436872802$

$-19.49471080717217$

$-13.95626752866798$

$-17.91855032286292$

$-15.85651725856067$

$-14.11357445534048$

$-15.95380094360344$

$-16.81463362680638$

$-17.52747659528578$

$-12.32544582148471$

$-14.94272030688530$

$-19.59583998973863$

$-17.96087839425457$

$-18.85185232648407$

$-20.22069907845570$

$-8.76757961355140$

$-7.48450527250298$

$-7.27194953245334$

$-8.39794355568520$

$-9.68386245787382$

$-9.88019278857905$

$-5.93074554816989$

$-5.06796932812490$

$-3.83639646105167$

$-3.43430921397742$

$-4.25801808582715$

$-5.48916913799315$

$-8.90754197535491$

$-6.62532272444943$

$-8.26917814843959$

$-10.54820260409021$

$-11.19752130206186$

$-5.38695978165796$
$-1.36585817007071$

$-0.57246253526014$

$-0.93446557368855$

0.89715591809706

1.49537555511580

$-3.37319473804549$

0.00562582281751

$-1.76154511537224$

$-1.83927927511464$

$-0.24922292346678$

0.64405044271436

1.24938070357500

$-3.40976132297419$

$-1.04180163736110$

3.03177689377079

1.40096944883704

2.29656542759644

3.56684426291822

10.32923578159822

8.83668234123485

10.48740064675392

8.06549511418948

6.49094548889752

8.14139231187549

5.85766402102190

7.32812806641306

10.46679327541359

8.97382539630081

7.35054980828082

8.80490940293495

6.43877262372990

4.18756828718828

5.80559871103358

4.91435901703516

5.81828204807886

10.26175470382049

7.99709231751490

3.63933153569172

4.50789953155542

3.98851699031453

2.88328976958428

2.33991699538388

2.85105143089380

3.92508038975034

2.27082611092021

2.12325625227073

1.46908044041866

0.93453005857243

1.11004224968182

1.76611565296407

5.36873697699772

4.45974740724666

1.46978446191740

2.40063863876702

4.42998763610528

2.49434918386932 
H 0.49027527399414

N -1.74303271762091

H -3.74428092612937

H -3.53298827327962

H 0.28270456586146

H -0.51986807530363

H $\quad-0.92445801993829$

H -2.58840681711875
$-3.18823478157833$

$-2.18687224004296$

$-3.93753288852044$

$-6.11903605265594$

$-11.26141449980265$

$-11.90426694160493$

$-1.99015901003096$

$-2.12953118802611$
1.35213114242460

0.24566263173562

0.72237932106839

1.89678668087650

4.99723777183342

3.68172045028817

$-0.34899630778254$

$-0.34003075606460$

Table SC22. Lowest-energy geometry from PES of the C12 Biphenyl Diamine conformation.

Au 2.91044190805856

Au 0.30976328543958

Au 1.43597204444793

Au 1.07650534964327

$\mathrm{Au}-1.64814720004187$

$\mathrm{Au}-0.47636028736327$

Au -0.82088219913928

Au -2.28352276358317

$\mathrm{Au}-0.14052700956243$

Au -1.26565678755318

Au -4.05945395593676

Au -3.02932931930624

$\mathrm{Au}-3.44780276098595$

Au -3.72455376725172

Au -4.97614420048721

Au -4.41977053185293

Au - 2.57213221625768

Au -1.84269117556278

Au -2.22588917151515

Au -2.86126240349710

Au -0.34677552116488

Au -0.87606581110806

Au 0.06487158043174

Au -2.67742500292110

Au -3.28543481029727

Au -2.31385407042950

$\mathrm{Au}-4.86675021354350$

Au - 1.45501988325856

Au 0.31617808136367

Au -0.46521215745871

Au -1.20355683269195

Au -0.33629178277309

Au -2.97199761103228

Au -5.51514247405484

$\mathrm{Au}-2.11928283335097$

Au -3.89441756378678

$\mathrm{Au}-4.57023900667125$

Au 0.42947238668767

$\mathrm{Au}-2.12156741633447$

$\mathrm{Au}-7.00161832386127$

C -1.90256798393747

C -1.87002263349777

C -2.79864419242662

C -3.76131842797022
4.12582487147253 4.05117013792159

2.07783112091871

4.97440632524131

4.87771707318809

2.90186892544099

5.67738842222063

3.92152679762988

0.19891333862415

1.95077968217267

1.82654194631806

0.06062468864751

2.68415133525952

5.45856735184350

3.74959705006510

4.67713762765987

3.52302487150367

$-1.60455898620256$

0.93759776132177

6.23623809713040

$-15.72432389601146$

$-13.93986252313050$

$-16.76144579299067$

$-16.25967554800198$

$-14.44182490196969$

$-17.30402871560873$

$-16.73189860673128$

$-12.35299195229583$

$-17.67630177241593$

$-14.96187308549257$

$-13.29966063870934$

$-15.83069497290115$

$-15.50578815523403$

$-14.93643070732125$

$-10.82075981506999$

$-12.87792107036550$

$-17.76319546956088$

$-18.48696202367946$

$-18.20552574825480$

$-17.15699008838252$

$-6.70443940486503$

$-5.81255935131685$

$-5.90734493532260$

$-6.94390769315656$
$-1.42083593623299$

$-2.30995540284467$

$-0.32536563144428$

0.44899943331775

$-0.39235713795464$

1.64867463999403

2.22052180239524

$-3.00450357203772$

0.76871086720046

$-1.19855146529908$

$-1.79643113393781$

$-0.02886585078491$

0.85461327925838

1.47485722262896

$-3.53477013673310$

$-0.99678559681673$

3.45360209520458

1.94307751012902

2.77264354207002

3.96191668739512

11.58519250416323

9.55951132938548

9.06855941014167

10.22818944153012

8.11275006617764

7.59928390230896

8.73865748659620

7.47037132763716

6.55071704664675

6.91120407684985

4.68013852545620

4.21665740267528

5.20694439498022

6.40303266134423

5.26349042777865

5.80245226847842

5.92067046682378

3.93499044519741

4.89643374379665

7.05953854838878

4.67065161235232

3.59189281927117

2.52887964178301

2.58975774472258 


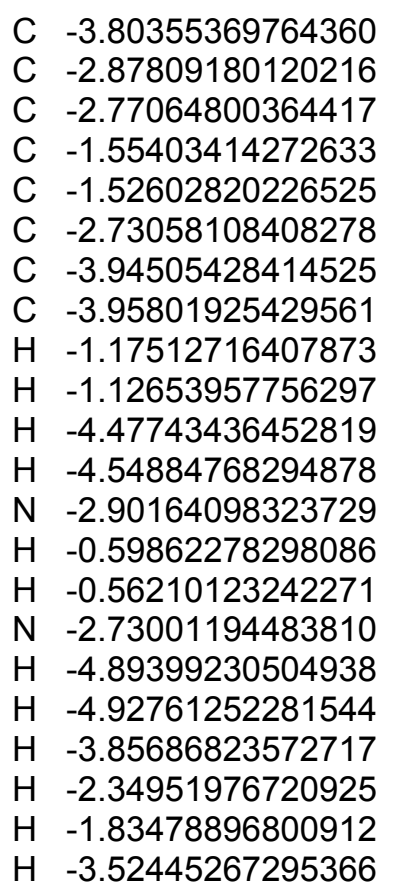

$-7.83905334204775$

$-7.71732846551254$

$-4.94868082486382$

$-4.38061679876190$

$-3.44017755978411$

$-3.03619951757601$

$-3.61364346673238$

$-4.54828315166839$

$-6.59897049123938$

$-4.99910170863159$

$-7.07295188885738$

$-8.65192222000305$

$-8.65886028728694$

$-4.70480277448845$

$-3.03622764032069$

$-1.95922136557139$

$-3.28672137485235$

$-4.94612655757366$

$-8.87900421593638$

$-8.34915447164549$

$-1.71741769404511$

$-1.88421546594553$
3.66341253368603

4.71841099611150

1.40211585083912

0.94931038889390

$-0.09832710736004$

$-0.72774090568903$

$-0.29816863090312$

0.74211708951314

5.49309498388453

3.59691662783972

1.76267188596215

3.67732638664975

5.79637616498559

1.39115700577244

$-0.44884363904175$

$-1.62312771496895$

$-0.75429823000880$

1.08132784052941

6.11510553186803

6.60888427842116

$-2.06258984911451$

$-2.26715161815825$

Table SC23. Lowest-energy geometry from PES of the TT11 dimeric Biphenyl Diamine conformation.

Au 2.43152611916513

Au 1.22725042605878

Au 1.14004135003357

Au -0.30752883978757

Au -1.61992398169908

$\mathrm{Au}-1.70831523709432$

Au -2.99593689679180

$\mathrm{Au}-0.08512372864256$

Au -0.26334328698667

Au -0.12238753114345

Au -1.63615159816450

Au -1.72420841159322

Au -3.21734827925433

$\mathrm{Au}-4.46323730944123$

Au -1.54911597048655

Au -3.07956845031672

$\mathrm{Au}-4.55560673158680$

Au -1.81446755992797

$\mathrm{Au}-3.25157954405874$

$\mathrm{Au}-5.73647163270261$

Au -5.20048926145690

$\mathrm{Au}-4.27976495770838$

Au -2.63553347341244

Au -5.02955716468074

$\mathrm{Au}-4.09184853978169$

$\mathrm{Au}-2.37876846106649$

Au -4.71809661854076

$\mathrm{Au}-3.25972122007116$

$\mathrm{Au}-0.16206469714358$
6.35217422297761

5.66361969303721

3.92033131378226

6.57279501910500

5.81814739220617

4.09203257627196

6.63532695008320

4.90531603133941

1.62619680373411

3.15061565163285

2.28519146262272

0.77147678337432

3.22382562206419

5.79086689137930

4.04496415652588

4.97140972472215

4.05775948473701

$-0.66413057721286$

1.70477677775452

6.54170114068950

$-17.92923747244566$

$-16.08824358384516$

$-18.43132096341382$

$-18.96937969295385$

$-17.14064305498507$

$-19.46701533671001$

$-19.87741977203445$

$-14.46988135924492$

$-18.85207118468606$
1.64202627260132

$-0.73369194656160$

1.74036566286341

1.68929507251209

$-0.73117404122666$

1.79484813451060

1.62758057992939

$-2.95756731426553$

1.69112622242105

$-0.71606601255526$

$-2.95470497370490$

$-0.69983309491749$

$-0.79253896689209$

$-0.87261457344343$

$-5.10725383776857$

$-3.03227672179958$

1.60317344160024

1.60425997600308

1.61374850008964

1.44754155909328

11.84448098487566

9.96527219004602

11.02688758770287

9.31724533310133

7.34465231695035

8.37856475675397

6.80449293220789

8.02488670979182

10.05411011937464 
Au -1.63104651977681

$\mathrm{Au}-0.54596881361951$

Au 0.94871871115069

Au -1.24552641494047

$\mathrm{Au}-3.61820247066413$

Au -2.18419717976778

$\mathrm{Au}-2.95546944345789$

Au -1.95434851673446

Au 2.30168070847958

Au 0.20101238881854

$\mathrm{Au}-4.24587445004086$

C -4.17687863850165

C -4.05073490861178

C -2.84513927308999

C -1.79075394167591

C -1.91088923390717

C -3.09536758469632

C -2.65272927504110

C -3.39738079955400

C -3.15191195950871

C -2.14169468107814

C -1.42118066515525

C -1.67355506492512

H -5.12340296498333

H -4.91960946722271

H -0.82369080344922

H -1.04905080468906

N -3.12811746817720

H -4.15981934869757

H -3.72782277652249

N -1.83318982867890

H -0.65716475619889

H -1.10069032356129

H -2.61645045268269

H -4.06399498012102

H -2.46871550564469

H -0.84260762362992

C -1.78891607714432

C -1.42116470819072

C -0.11331699005524

C 0.82521430403188

C 0.46251372293977

C -0.85737796961791

C 0.24304154107415

C -0.32880101770206

C -0.04893142846357

C 0.80957113152520

C 1.42712463458624

C 1.14403548007094

H -2.81932844756967

H -2.18027784172802

H 1.86660087205994

H 1.21024644282193

N -1.26458547601204

H -1.00298325749418

H $\quad-0.52843320787128$
$-16.59034861621919$

$-14.99566482059840$

$-17.03625802045912$

$-17.63281488552768$

$-18.05885549511996$

$-12.98374596631268$

$-15.53137560491515$

$-20.35042981876142$

$-19.22951204700673$

$-19.86699109590389$

$-20.70071682560204$

$-9.78224167093419$

$-8.39073395389793$

$-7.73150271587213$

$-8.53276350671478$

$-9.91978401485028$

$-10.57013501918006$

$-6.28229411512023$

$-5.60046142293989$

$-4.25691078932931$

$-3.54897798691407$

$-4.19514705136902$

$-5.53624897623882$

$-10.26645367778717$

$-7.80511455962414$

$-8.07131335854307$

$-10.51572551940145$

$-11.98142027783187$

$-6.14100447776453$

$-3.76320437137151$

$-2.18857901719217$

$-3.63427834352063$

$-6.01008382712574$

$-12.47863829397943$

$-12.38518119236906$

$-1.82526374070885$

$-2.06682911400762$

$-9.50583347553615$

$-8.15951987141299$

$-7.72949980179816$

$-8.71921569277257$

$-10.06930614692597$

$-10.46821667333269$

$-6.29138639546514$

$-5.39516506041883$

$-4.02603834578578$

$-3.49597321137148$

$-4.38316052985157$

$-5.75266393155554$

$-9.81523499864500$

$-7.41324119291652$

$-8.43038628471408$

$-10.82115104729317$

$-11.83400887118609$

$-5.78110392260270$

$-3.35051812338156$
9.10242118494856

7.01433174486941

7.92132150340952

6.19598261979635

4.66534227541600

6.04277107742018

5.28818795984876

5.69780282859131

8.88530907293643

7.23160276240524

4.21985457720963

2.34569385622984

2.43203226064133

2.09744583975339

1.59855446703878

1.49169057359548

1.90103745096631

2.33850505160344

3.32967198531967

3.63634271126299

2.96119130929883

1.93991563034643

1.63985748097933

2.64029318525850

2.77171287309522

1.34725583267419

1.14733573587129

1.99883257630982

3.91229130812178

4.43726858285158

3.29722271948454

1.37687569784544

0.82786023306200

1.25861179661615

2.12839116209007

4.02241882188598

3.63775192190796

4.88732660678463

4.96209221503756

4.63218585141678

4.26077052630973

4.17468122457645

4.45602308799416

4.64268123171813

5.57538349153970

5.53983117993154

4.55254660705452

3.64733182198351

3.69570197081129

5.12497827106607

5.24277761476854

4.04550672059322

3.86939918098325

4.29000322233195

6.35648181447945

6.26887415722317 

N 0.90954118599777
H 2.10122461960144
H 1.60166963011493
H -0.46586390724193
$-2.09433636186544$
$-3.98911325918954$
$-6.41562188165027$
$-12.42978796993897$
4.38555570977481
2.86803768740546
2.94378675835508
$-11.95331519519775$
4.02475938319364
H -2.00338905169310
$-1.54836365778409$
3.54732000258315
H 0.88519369514083
$-1.78003773944077$
5.25623649515733
3.78038830030694

Table SC24. Lowest-energy geometry from PES of the CT11 dimeric Biphenyl Diamine conformation.

Au 3.03482520525430

Au 1.11067362379821

Au 1.57291336360156

Au 0.47696566573419

Au -1.56629908958452

$\mathrm{Au}-1.09197005943165$

$\mathrm{Au}-2.08436836649162$

$\mathrm{Au}-0.88178438469310$

Au -0.01689821226424

$\mathrm{Au}-0.44507016237744$

$\mathrm{Au}-2.63937776593436$

Au -2.21567233791296

Au -3.38277990984164

$\mathrm{Au}-4.30987632199552$

$\mathrm{Au}-3.00766605909295$

$\mathrm{Au}-3.73267286384877$

$\mathrm{Au}-3.85304220156430$

$\mathrm{Au}-1.76084265199134$

Au -2.87039781673705

Au -4.74878851936245

$\mathrm{Au}-4.10683096468749$

Au -3.52780512762984

Au -1.89730533578314

Au -4.69292741185959

Au -4.12217085248280

Au -2.43239336834173

Au -5.12427621118001

Au -2.89276385064125

Au 0.19172055343595

Au -1.24617036105069

Au -0.59585163113351

Au 0.88601389897021

Au -1.72144304456023

$\mathrm{Au}-4.43599625266369$

Au -2.23782208841905

Au -3.39585214354603

Au -2.78971255518017

Au 2.22875363401896

Au -0.27762053742680

$\mathrm{Au}-5.40538190958050$

C -4.43717602964545

C -4.09756007491550

C -2.77219564199373

C -1.79984383843018
7.64493473283570

7.14094680192994

5.36788064932047

8.15274994123200

7.60496157679446

5.83102841791911

8.49852444154957

6.58109892036184

3.23772393444524

4.78771269163130

4.14161173261373

2.57858984439031

5.18352461627631

7.87178501162876

5.92949200368928

6.96544482586099

6.09958202869113

1.12268383746005

3.62263294743989

8.69546810692227

$-19.52579122088211$

$-17.74954993689522$

$-20.25208706930986$

$-20.53385113901852$

$-18.76510158282026$

$-21.25382948862757$

$-21.42022186972346$

$-16.19870399466317$

$-20.88131518742894$

$-18.47454301209603$

$-16.94090169009492$

$-19.12605187265735$

$-19.49628865800694$

$-19.67236427274739$

$-14.77056041894809$

$-17.23109477703476$

$-22.12789810949027$

$-21.45379649639618$

$-21.87176010160047$

$-22.23397214524526$

$-7.50285333592197$

$-6.16296522702501$

$-5.76788334726339$

$-6.79229070365671$
$-0.32928167067395$

$-2.23100556414813$

0.16871345880928

0.53888760451667

$-1.37492747916464$

1.07693811609843

1.28438523511931

$-3.95065319032033$

0.56637316469720

$-1.78354287592379$

$-3.45829243687634$

$-1.27942346397129$

$-0.92892827198796$

$-0.65418951772758$

$-5.55225041917778$

$-3.12144671962747$

1.74763525931587

0.96050646911187

1.39679293033895

1.93603544030659

13.09162192205921

11.03060509958281

11.64219717954448

10.61159209206227

8.45361779103533

9.02231816736372

8.10820260218758

8.89054856370976

10.06696199375989

9.51496091320902

7.20616555018967

7.71041490351135

6.61409704403040

5.75017359421453

6.68652461163962

6.16931474536538

6.33129216420274

8.30632585921396

7.25730311177291

5.49431701076748

1.54047926420912

1.74582960498927

2.04645649870490

2.13224762377492 


\begin{tabular}{|c|c|c|c|}
\hline & -2.12480241425873 & -8.13423128089412 & \\
\hline & -3.45401549610661 & -8.52014009525455 & 1.62362450634575 \\
\hline & -2.41962060109605 & 3180714626101 & \\
\hline & 6127455326359 & & \\
\hline & .03246271212465 & & \\
\hline & .73124222385770 & -1.61216268012559 & \\
\hline & .77772102240191 & -2.51702588644680 & \\
\hline & .12170022531346 & & \\
\hline & .47956047775685 & & \\
\hline & -4.88469901299413 & & \\
\hline & & & \\
\hline & 34 & & \\
\hline & 60 & & \\
\hline & 644 & & \\
\hline & 6617 & -1. & 3 \\
\hline & 5928 & & \\
\hline & & & \\
\hline & & & \\
\hline & & & \\
\hline & -4.6 & & \\
\hline & -1.9 & & \\
\hline & -0.4 & & \\
\hline & -2.7 & -11 & \\
\hline & & & \\
\hline & & & \\
\hline & & & \\
\hline & & & \\
\hline & -1.6 & -12 & \\
\hline & -1.4 & & \\
\hline & -2.5 & & \\
\hline & -2.4 & & \\
\hline & -1.2 & & \\
\hline & & & \\
\hline & & & \\
\hline & & & \\
\hline & -3.4 & & \\
\hline & 0.46 & -9.6 & \\
\hline & 0.32 & -12 & \\
\hline & $-1.7 c$ & & \\
\hline & & & \\
\hline & & & \\
\hline & & & \\
\hline & & & \\
\hline & & & \\
\hline & & & \\
\hline & & & \\
\hline & & & \\
\hline & & & \\
\hline
\end{tabular}

Table SC25. Lowest-energy geometry from PES of the CC11 dimeric Biphenyl Diamine conformation.

Au 3.00631621590877

Au 1.13658757603748

Au 1.55250971231363
7.68851184966334 7.16390413297472 5.39794274107932
$-0.21003107347776$

$-2.16057119318966$

0.25181311527703 
Au 0.42136258416090

$\mathrm{Au}-1.56629899955817$

Au -1.13943069856626

Au -2.16143870309961

Au -0.80547755856545

$\mathrm{Au}-0.02643216918971$

Au -0.40967957536089

$\mathrm{Au}-2.55331730396319$

$\mathrm{Au}-2.17244335605484$

$\mathrm{Au}-3.37071500969274$

$\mathrm{Au}-4.32976606775761$

Au - 2.88328186043916

$\mathrm{Au}-3.68121347743397$

Au -3.91856711637537

Au -1.76084299588551

Au -2.90469185014768

$\mathrm{Au}-4.84291046044033$

$\mathrm{Au}-3.62744896484112$

Au -3.19539159418233

$\mathrm{Au}-1.60162661445371$

$\mathrm{Au}-4.49545399430674$

$\mathrm{Au}-4.08088220604419$

Au -2.43239299823488

Au -5.20703121360999

Au - 2.72592737376227

Au 0.29484034582909

$\mathrm{Au}-1.10502185154682$

Au -0.63308978532298

Au 0.81156779832643

$\mathrm{Au}-1.90489352332715$

Au -4.69651375255226

$\mathrm{Au}-2.23782200357076$

$\mathrm{Au}-3.53186123457917$

$\mathrm{Au}-3.08974673277930$

Au 2.12318835237039

Au -0.48965561992423

$\mathrm{Au}-5.77891518037891$

C -3.98551156465540

C -3.73394937728240

C -2.44049719304349

C -1.40652080911221

C -1.64473935968472

C -2.94257056075348

C -2.18016051228866

C -3.18565016340823

C -2.94108739928365

C -1.66372905905361

C -0.64823578212989

C -0.90825135681814

H $\quad-5.00440768154786$

H -4.56801036626741

H -0.39466819060141

H -0.82122648849052

N -3.16928005271812

H -4.18235097122757

H $\quad-3.75001551560652$
8.17217920297500

7.60496194556966

5.83637682451662

8.49453655563585

6.58446847168076

3.25311542267753

4.79643946756619

4.12925437245294

2.57104273925865

5.16599631211597

7.84567196270391

5.91312108661879

6.94281516112096

6.08032241443676

1.12268406189833

3.61216500033684

8.66733085624770

$-19.48403673380385$

$-17.72207424217463$

$-20.28147575128837$

$-20.45997160281523$

$-18.70407717776316$

$-21.25382892027401$

$-21.31900615130712$

$-16.18639306800962$

$-20.97708798972869$

$-18.51943128426275$

$-17.00149518298763$

$-19.23950867391696$

$-19.51164403731887$

$-19.58530397009041$

$-14.77056008823540$

$-17.18457922535008$

$-22.10206786599712$

$-21.61558160379549$

$-21.93965606566152$

$-22.11007864510509$

$-7.66416849016857$

$-6.30211511920813$

$-5.81979071496385$

$-6.78006164246455$

$-8.14222844639540$

$-8.61701709627252$

$-4.38085374813900$

$-3.50802243179723$

$-2.14504702634622$

$-1.60815199837813$

$-2.45401374127827$

$-3.81213031155405$

$-8.00125655053918$

$-5.59270724405731$

$-6.45951258251132$

$-8.86413854262406$

$-9.96849838944736$

$-3.90921325948174$

$-1.49195668402406$
0.59132231606185

$-1.37492697977280$

1.09031982190938

1.26951413942696

$-3.93068295274865$

0.61091423494729

$-1.75079725746700$

$-3.48210454684445$

$-1.29155318320779$

$-0.97303989839500$

$-0.72521242365126$

$-5.58723376814674$

$-3.17572707376023$

1.68919911179120

0.96050598025171

1.36681147874850

1.85106416361904

13.23921702394675

11.13276669911014

11.57511932337802

10.83005006949783

8.62640077150883

9.02231797146504

8.38161930871540

8.94123070620400

9.79779985782988

9.39363981016601

7.03114248287040

7.38459098241714

6.55533378821109

5.96669178069994

6.68652502984459

6.28210407400547

6.37929235552094

7.84109546257020

7.04850717816750

5.80768732853148

1.54730815776007

1.73538327770753

2.04638673316252

2.15642463114246

1.97516019774466

1.66169333762762

2.25993140011938

2.74417832777949

2.95941463274056

2.70656270199944

2.22016328188104

2.00262734455555

1.29089666756523

1.60941260295144

2.44675293380396

2.10103666660530

1.43584338296411

2.98559772325513

3.32900124853317 


$\begin{array}{clcc}\mathrm{N} & -1.41945624928789 & -0.20639819237620 & 2.87403004502418 \\ \mathrm{H} & 0.34764007212112 & -2.03988819505872 & 1.98736826684340 \\ \mathrm{H} & -0.10380462218027 & -4.44527008004828 & 1.59725454231892 \\ \mathrm{H} & -4.14407085696749 & -10.27850032611097 & 1.47231633561921 \\ \mathrm{H} & -2.52226534517634 & -10.61385993544958 & 1.89927494552292 \\ \mathrm{H} & -2.03891226300252 & 0.22749176443009 & 3.57342988524422 \\ \mathrm{H} & -0.44301197312720 & 0.00367892520736 & 3.12619053325644 \\ \mathrm{C} & -2.80349833502287 & -11.29694574346904 & 4.57349924887014 \\ \mathrm{C} & -2.69670554083541 & -9.90984721307135 & 4.73678325930032 \\ \mathrm{C} & -1.50247762359384 & -9.30060716465485 & 5.19360028243513 \\ \mathrm{C} & -0.42035930163290 & -10.16550300088903 & 5.49761884177453 \\ \mathrm{C} & -0.51137723928052 & -11.55144748533393 & 5.33010731074971 \\ \mathrm{C} & -1.70304882545531 & -12.12874862345633 & 4.85168764684151 \\ \mathrm{C} & -1.38416403417231 & -7.83179876732145 & 5.31457561589367 \\ \mathrm{C} & -2.52359382525630 & -7.00081220177159 & 5.44293611457301 \\ \mathrm{C} & -2.42080074565163 & -5.61076538584391 & 5.48579350323151 \\ \mathrm{C} & -1.15907875252810 & -4.97064129958429 & 5.40059316077826 \\ \mathrm{C} & -0.01052446472744 & -5.79215987235356 & 5.29059948488421 \\ \mathrm{C} & -0.12724827676949 & -7.18398785943431 & 5.25113993549334 \\ \mathrm{H} & -3.75145421103532 & -11.73817619425982 & 4.22013105996615 \\ \mathrm{H} & -3.55761063093482 & -9.28108166914129 & 4.46569007472771 \\ \mathrm{H} & 0.51619583226746 & -9.74745832402241 & 5.89796938460555 \\ \mathrm{H} & 0.34587419852018 & -12.19522372071880 & 5.58987597199973 \\ \mathrm{~N} & -1.81051922409442 & -13.55256404356729 & 4.72070006208503 \\ \mathrm{H} & -3.52555370334791 & -7.45128936570806 & 5.51951048429342 \\ \mathrm{H} & -3.33134119077519 & -4.99727304565916 & 5.58547911428722 \\ \mathrm{~N} & -1.05547384462654 & -3.58755679498981 & 5.46154183699278 \\ \mathrm{H} & 0.98579035651525 & -5.32411651441962 & 5.21614157023300 \\ \mathrm{H} & 0.79041548151600 & -7.77924852058708 & 5.11814407063190 \\ \mathrm{H} & -0.93425883553337 & -13.98878233015065 & 4.39809548532649 \\ \mathrm{H} & -2.56758091083202 & -13.83668147520585 & 4.08272955060412 \\ \mathrm{H} & -1.88205420200619 & -3.07563895796131 & 5.13310710909529 \\ \mathrm{H} & -0.19256810698040 & -3.19792387013250 & 5.07117398465038\end{array}$

Table SC26. Lowest-energy geometry for $\mathrm{N}_{2} \mathrm{C}_{12} \mathrm{H}_{12} \cdot \mathrm{N}_{2} \mathrm{C}_{12} \mathrm{H}_{12}$.
C -4.23009751680479
C -4.09908773568471
C -2.87455103999170
C -1.80296368195847
C -1.92751387360764
C -3.13516684032239
C -2.69612537684316
C -3.50829493267369
C -3.29312085297518
C -2.23715758558493
C -1.44154786877726
C -1.66504355906612
H $\quad-5.19612396104391$
H -4.98123084992047
H -0.81812992360956
H -1.05061894440256
N -3.21173709240676
H -4.30597759706318
H $\quad-3.92619004327411$
$-9.75524025788599$
$-8.36546697117038$
$-7.70725042486461$
$-8.51769188444733$
$-9.90389359090030$
$-10.55337952845189$
$-6.26056512301441$
$-5.57282351584470$
$-4.22909076675806$
$-3.49756411949300$
$-4.16637917203457$
$-5.50854663965004$
$-10.23301227617454$
$-7.77669439583770$
$-8.06435895558286$
$-10.50242343848925$
$-11.96242781198404$
$-6.11490125108481$
$-3.74287898528774$

2.29948225823683

2.40288392719297

2.13833500837357

1.69155578875496

1.56709996495137

1.90525854016628

2.39727000298645

3.33093040645326

3.65237124760867

3.05662566082990

2.09449682386864

1.78450714137570

2.53946421385781

2.70125606403619

1.50164860291521

1.26771576575284

1.95436608781218

3.86450945672360

4.41510202282965 


$\begin{array}{llll}\mathrm{N} & -1.98633423565730 & -2.16792112304871 & 3.38384389600185 \\ \mathrm{H} & -0.62428373671613 & -3.61762252496018 & 1.59948414618377 \\ \mathrm{H} & -1.01611773599834 & -5.98569044465601 & 1.03284389549479 \\ \mathrm{H} & -2.57772485486000 & -12.44087967987997 & 1.30340251341141 \\ \mathrm{H} & -4.16495945367621 & -12.33579354093278 & 1.87495139732007 \\ \mathrm{H} & -2.47844550128697 & -1.83177208515017 & 4.21718295795211 \\ \mathrm{H} & -0.98862470120501 & -1.89642257899959 & 3.38488737195796 \\ \mathrm{C} & -1.71338879679834 & -9.55700999963879 & 4.85546192678764 \\ \mathrm{C} & -1.36667661621734 & -8.20638187676576 & 4.91750226875173 \\ \mathrm{C} & -0.06074858013006 & -7.75350920850946 & 4.60652559566075 \\ \mathrm{C} & 0.89428019356742 & -8.74195941861975 & 4.27228337048193 \\ \mathrm{C} & 0.55607136359049 & -10.09746042653608 & 4.19902888699786 \\ \mathrm{C} & -0.76753027738311 & -10.53241265629418 & 4.45406506303359 \\ \mathrm{C} & 0.27505700884729 & -6.31191142611052 & 4.58664386168506 \\ \mathrm{C} & -0.37196073315228 & -5.38331666982713 & 5.43636008572003 \\ \mathrm{C} & -0.08958038987849 & -4.01529155531342 & 5.38305565672485 \\ \mathrm{C} & 0.84843320122736 & -3.50765375919611 & 4.45878718450900 \\ \mathrm{C} & 1.52887367450084 & -4.42385840192581 & 3.62958320944604 \\ \mathrm{C} & 1.24259977610597 & -5.79216531492682 & 3.69450195709683 \\ \mathrm{H} & -2.75104215635613 & -9.86667441107344 & 5.05942056604413 \\ \mathrm{H} & -2.14952523947750 & -7.46924202045581 & 5.15625284729792 \\ \mathrm{H} & 1.93609426929719 & -8.44399614126116 & 4.06784665042349 \\ \mathrm{H} & 1.32453729713882 & -10.83792471709428 & 3.91487451834596 \\ \mathrm{~N} & -1.13350502678271 & -11.87290825670071 & 4.32704790451268 \\ \mathrm{H} & -1.11127680963622 & -5.74417185569255 & 6.16867863735202 \\ \mathrm{H} & -0.63154909099135 & -3.32011863280063 & 6.04692551938311 \\ \mathrm{~N} & 1.00765722511217 & -2.11276897973473 & 4.29179095979593 \\ \mathrm{H} & 2.26349225965351 & -4.05263323886480 & 2.89380013497360 \\ \mathrm{H} & 1.75677279096990 & -6.47384582369198 & 2.99770072191367 \\ \mathrm{H} & -0.42639271222677 & -12.45600466379968 & 3.86751270494254 \\ \mathrm{H} & -2.06495135222042 & -12.03230636902964 & 3.91051886810231 \\ \mathrm{H} & 0.87275740172899 & -1.57232994881782 & 5.15529740067588 \\ \mathrm{H} & 1.88669381492135 & -1.84394314073530 & 3.83378133629340\end{array}$




\section{Experimental Analysis Procedures}
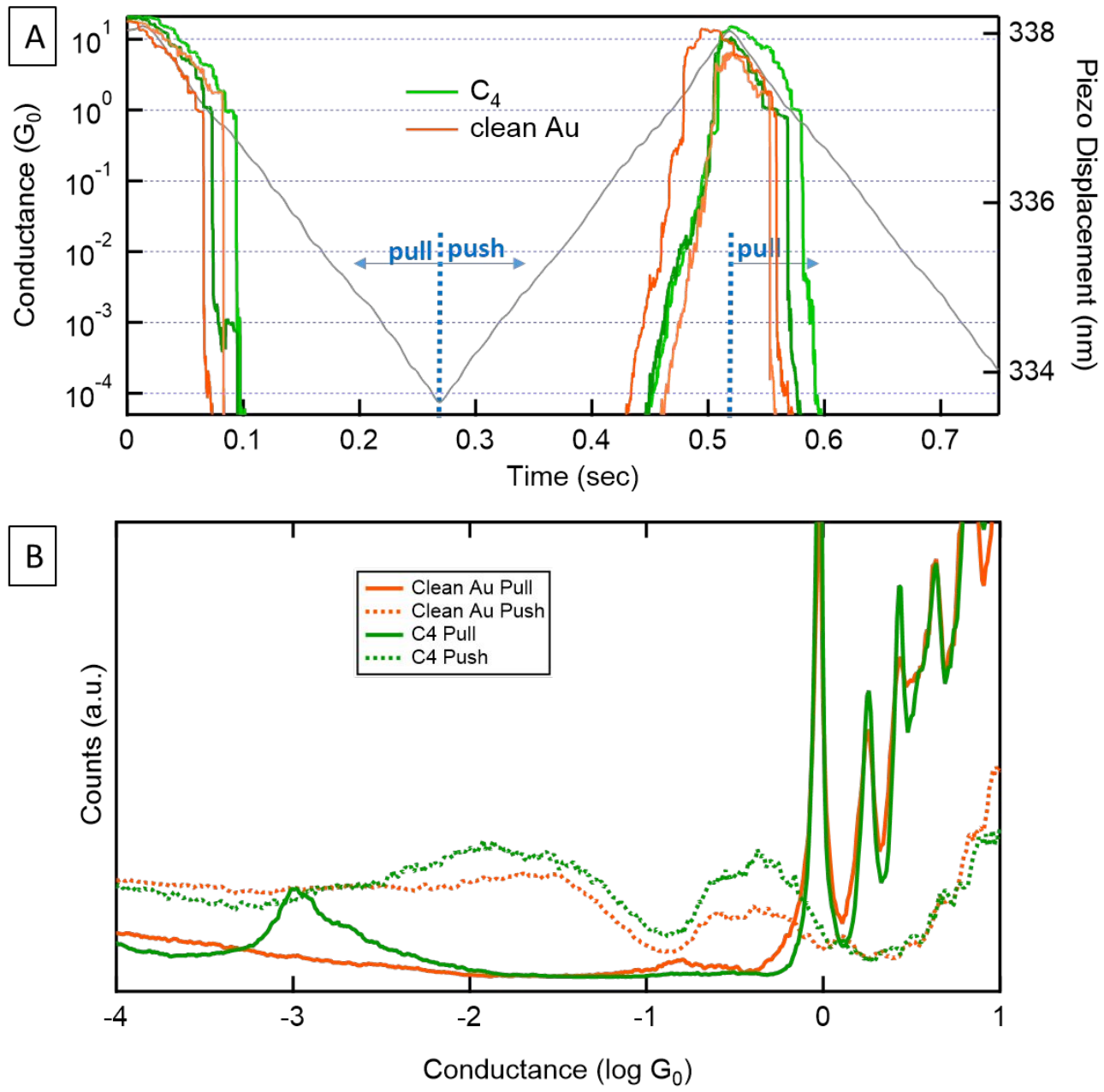

Figure SC10. A) Sample pull-push traces recorded on clean gold (orange) and on $\mathrm{C}_{4}$ (green). The grey trace shows the position of the piezo nano-positioner which moves the sample relative to the tip. The pull traces are binned separately from the push traces to create histograms shown in (B). (B) Log-binned conductance histograms created from at least 3000 pull (solid lines) and push (dashed lines) traces. Push traces and histograms do not show features at integer values of $\mathrm{G}_{0}$.

To measure snap-back and construct our 2D histograms that relate junction conductance directly to junction geometry, we successively stretch and squeeze our junctions in ambient conditions in the presence of diamine molecules. Sample traces measured both on clean gold and in the presence of $\mathrm{C} 4$ diamine demonstrating this procedure are shown in Figure SC10A. We show 
histograms constructed from thousands of pulling and pushing traces measured both on clean gold and on a diamine molecule in Figure SC10B. The histograms make clear that no reproducible conductance signatures at integer multiples of $\mathrm{G}_{0}$ are present when the junction is squeezed together both on clean gold and in the presence of molecules. Instead, broad features that span $\sim 0.1 \mathrm{G}_{0}$ to $1 \mathrm{G}_{0}$ conductance range are observed. Such fractional $\mathrm{G}_{0}$ features measured on noble metal surfaces at room temperature have previously been attributed to the adsorption of molecular hydrogen or oxygen and we hypothesize that in ambient conditions, these and other small molecules can bind on the blunted electrodes following snap-back, reducing the conductance of the metal-metal contacts upon pushing. ${ }^{14-17} \mathrm{We}$ observe that following the features below $\sim 1 \mathrm{G}_{0}$, the trace conductance jumps to many times $1 \mathrm{G}_{0}$, indicating a large metallic contact forms once the blunt electrodes merge during pushing. In contrast, upon pulling, a pristine metal surface is exposed during plastic deformations prior to rupture, resulting in a sharp single atom contact with $\sim 1 \mathrm{G}_{0}$ conductance. Through snap-back, this protruding contact dissipates energy and relaxes to a blunt geometry. Based on these observations and earlier published work, we use $0.5 \mathrm{G}_{0}$ as the conductance at which metallic contact breaks upon pulling and $0.1 \mathrm{G}_{0}$ to identify the formation of the metallic contact upon pushing. ${ }^{18,19}$ These cutoffs were used for construction both of the snapback distributions and the 2D histograms.

By comparing the displacement when the $\mathrm{G}_{0}$ contact is ruptured (at $0.5 \mathrm{G}_{0}$ ) and when it is reformed (at $0.1 \mathrm{G}_{0}$ ), we can measure how much the junction relaxes following rupture. These 
snap-back distribution for clean gold contacts constructed using the procedure outlined above are shown in Figure 1E. Snap-back distributions measured on junctions with molecules using the same procedure are shown in Figure SC11. We observe that aside from fluctuations around a mean value of $\sim 0.8 \mathrm{~nm}$, no significant change in snap-back distributions is observed in the presence of diamine molecules, indicating that they do not change the mechanical properties of the electrodes unlike stronger-bound molecules such as thiols.

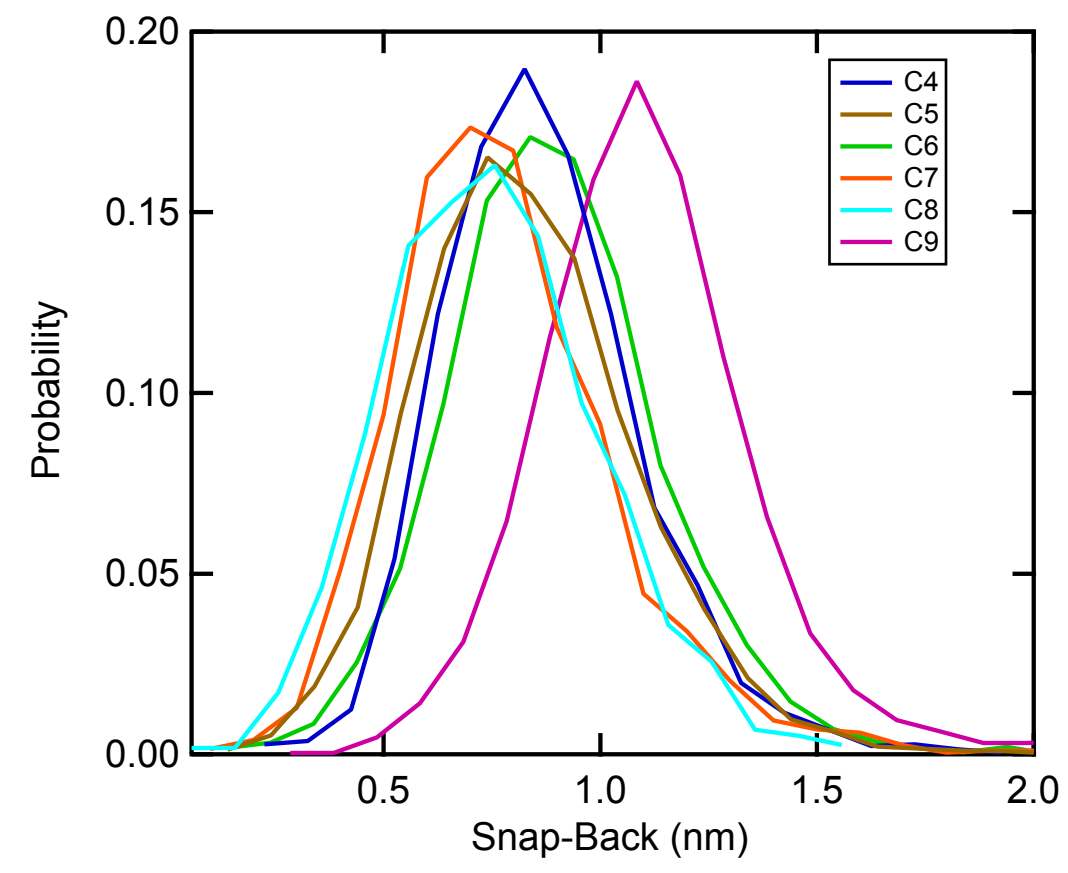

Figure SC11. A) Snap-back distributions constructed from thousands of traces measured in different experiments in the presence of diamine alkane molecules of varying lengths. 


\section{Voltage Dependence}

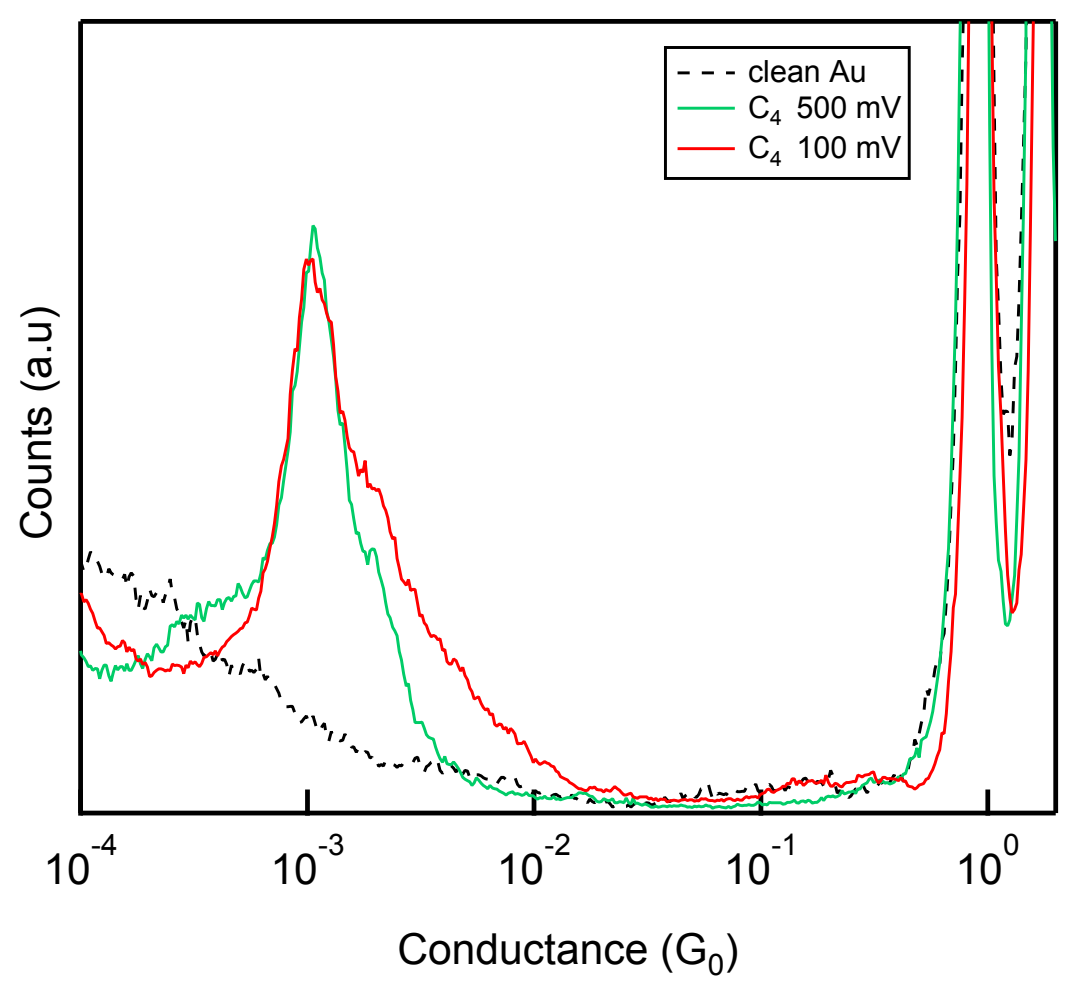

Figure SC12. Log-binned conductance histograms of $\mathrm{C}_{4}$ Diamine measured at 100 and $500 \mathrm{mV}$. The position of the diamine single-molecule conductance peak does not depend on bias up to $\sim 1000 \mathrm{mV}$ for all molecules in our sample.

\section{References}

(1) Neese, F. The ORCA Program System. Wiley Interdiscip. Rev. Comput. Mol. Sci. 2012, 2 (1), 73-78. https://doi.org/10.1002/wcms.81.

(2) Neese, F. ORCA - An Ab Initio, DFT and Semiempirical SCF-MO Package, Ver. 4.0. Max Planck Institute for Chemical Energy Conversion: Mülheim a. d. Rurh, Germany 2017.

(3) Neese, F. Software Update: The ORCA Program System, Version 4.0. Wiley Interdiscip. Rev. Comput. Mol. Sci. 2018, 8 (1), e1327. https://doi.org/10.1002/wcms.1327.

(4) Becke, A. D. Density-Functional Exchange-Energy Approximation with Correct Asymptotic Behavior. Phys. Rev. A 1988, 38 (6), 3098-3100. https://doi.org/10.1103/PhysRevA.38.3098. 
(5) Perdew, J. P. Density-Functional Approximation for the Correlation Energy of the Inhomogeneous Electron Gas. Phys. Rev. B 1986, 33 (12), 8822-8824.

https://doi.org/10.1103/PhysRevB.33.8822.

(6) Weigend, F.; Ahlrichs, R. Balanced Basis Sets of Split Valence, Triple Zeta Valence and Quadruple Zeta Valence Quality for H to Rn: Design and Assessment of Accuracy. Phys. Chem. Chem. Phys. 2005, 7 (18), 3297-3305. https://doi.org/10.1039/B508541A.

(7) Grimme, S.; Antony, J.; Ehrlich, S.; Krieg, H. A Consistent and Accurate Ab Initio Parametrization of Density Functional Dispersion Correction (DFT-D) for the 94 Elements H-Pu. J. Chem. Phys. 2010, 132 (15), 154104. https://doi.org/10.1063/1.3382344.

(8) Grimme, S.; Ehrlich, S.; Goerigk, L. Effect of the Damping Function in Dispersion Corrected Density Functional Theory. J. Comput. Chem. 2011, 32 (7), 1456-1465. https://doi.org/10.1002/jcc.21759.

(9) Weigend, F. Accurate Coulomb-Fitting Basis Sets for H to Rn. Phys. Chem. Chem. Phys. 2006, 8 (9), 1057-1065. https://doi.org/10.1039/B515623H.

(10) Adamo, C.; Barone, V. Toward Reliable Density Functional Methods without Adjustable Parameters: The PBE0 Model. J. Chem. Phys. 1999, 110 (13), 6158-6170. https://doi.org/10.1063/1.478522.

(11) Ganyushin, D.; Gilka, N.; Taylor, P. R.; Marian, C. M.; Neese, F. The Resolution of the Identity Approximation for Calculations of Spin-Spin Contribution to Zero-Field Splitting Parameters. J. Chem. Phys. 2010, 132 (14), 144111. https://doi.org/10.1063/1.3367718.

(12) Kossmann, S.; Neese, F. Comparison of Two Efficient Approximate Hartee-Fock Approaches. Chem. Phys. Lett. 2009, 481 (4), 240-243. https://doi.org/https://doi.org/10.1016/j.cplett.2009.09.073.

(13) Neese, F.; Wennmohs, F.; Hansen, A.; Becker, U. Efficient, Approximate and Parallel Hartree-Fock and Hybrid DFT Calculations. A 'Chain-of-Spheres' Algorithm for the Hartree-Fock Exchange. Chem. Phys. 2009, 356 (1), 98-109. https://doi.org/https://doi.org/10.1016/j.chemphys.2008.10.036.

(14) Csonka, S.; Halbritter, A.; Mihály, G. Pulling Gold Nanowires with a Hydrogen Clamp: Strong Interactions of Hydrogen Molecules with Gold Nanojunctions. 2006. https://doi.org/10.1103/PhysRevB.73.075405.

(15) Barnett, R. N.; Ha, H.; Scherbakov, A. G.; Landman, U. Hydrogen Welding and Hydrogen Switches in a Monatomic Gold Nanowire. 2004. https://doi.org/10.1021/n1049054n.

(16) Aradhya, S. V.; Frei, M.; Halbritter, A.; Venkataraman, L. Correlating Structure, Conductance, and Mechanics of Silver Atomic-Scale Contacts. ACS Nano 2013, 7 (4), 3706-3712. https://doi.org/10.1021/nn4007187.

(17) Untiedt, C.; Dekker, D. M. T.; Djukic, D.; Van Ruitenbeek, J. M. Absence of Magnetically Induced Fractional Quantization in Atomic Contacts. https://doi.org/10.1103/PhysRevB.69.081401. 
(18) Kamenetska, M.; Quek, S. Y.; Whalley, A. C.; Steigerwald, M. L.; Choi, H. J.; Louie, S. G.; Nuckolls, C.; Hybertsen, M. S.; Neaton, J. B.; Venkataraman, L. Conductance and Geometry of Pyridine-Linked Single-Molecule Junctions. J. Am. Chem. Soc. 2010, 132 (19), 6817-6821. https://doi.org/10.1021/ja1015348.

(19) Quek, S. Y.; Kamenetska, M.; Steigerwald, M. L.; Choi, H. J.; Louie, S. G.; Hybertsen, M. S.; Neaton, J. B.; Venkataraman, L. Mechanically Controlled Binary Conductance Switching of a Single-Molecule Junction. Nat. Nanotechnol. 2009, 4 (4), 230-234. https://doi.org/10.1038/nnano.2009.10. 